\title{
Cardiac injury associated with severe disease or ICU admission and death in hospitalized patients with COVID-19: a meta-analysis and systematic review
}

Xinye $\mathrm{Li}^{1,2+}$, Xiandu Pan ${ }^{1,2+}$, Yanda $\mathrm{Li}^{3 \dagger}, \mathrm{Na} \mathrm{An}{ }^{4 \dagger}$, Yanfen Xing ${ }^{5}$, Fan Yang ${ }^{1}, \mathrm{Li} \mathrm{Tian}^{2}$, Jiahao Sun ${ }^{2}$, Yonghong Gao ${ }^{4}$, Hongcai Shang ${ }^{4^{*}}$ and Yanwei Xing ${ }^{1 *}$

\begin{abstract}
Background: Cardiac injury is now a common complication of coronavirus disease (COVID-19), but it remains unclear whether cardiac injury-related biomarkers can be independent predictors of mortality and severe disease development or intensive care unit (ICU) admission.

Methods: Two investigators searched the PubMed, EMBASE, Cochrane Library, MEDLINE, Chinese National Knowledge Infrastructure (CNKI), Wanfang, MedRxiv, and ChinaXiv databases for articles published through March 30, 2020. Retrospective studies assessing the relationship between the prognosis of COVID-19 patients and levels of troponin I $(\mathrm{Tnl})$ and other cardiac injury biomarkers (creatine kinase [CK], CK myocardial band [CK-MB], lactate dehydrogenase $[\mathrm{LDH}]$, and interleukin-6 [LL-6]) were included. The data were extracted independently by two investigators.

Results: The analysis included 23 studies with 4631 total individuals. The proportions of severe disease, ICU admission, or death among patients with non-elevated Tnl (or troponin T [TnT]), and those with elevated Tnl (or TnT) were 12.0\% and $64.5 \%, 11.8 \%$ and $56.0 \%$, and $8.2 \%$ and. 59.3\%, respectively. Patients with elevated Tnl levels had significantly higher risks of severe disease, ICU admission, and death (RR 5.57, 95\% Cl 3.04 to $10.22, P<0.001 ; \mathrm{RR} 6.20,95 \% \mathrm{Cl} 2.52$ to 15.29, $P<0.001$; RR 5.64, 95\% Cl 2.69 to $11.83, P<0.001$ ). Patients with an elevated CK level were at significantly increased risk of severe disease or ICU admission (RR 1.98, 95\% Cl 1.50 to 2.61, $P<0.001$ ). Patients with elevated CK-MB levels were at a higher risk of developing severe disease or requiring ICU admission (RR 3.24, 95\% Cl 1.66 to $6.34, P=$ 0.001). Patients with newly occurring arrhythmias were at higher risk of developing severe disease or requiring ICU admission (RR 13.09, 95\% Cl 7.00 to 24.47, P<0.001). An elevated IL-6 level was associated with a higher risk of developing severe disease, requiring ICU admission, or death.

(Continued on next page)
\end{abstract}

\footnotetext{
* Correspondence: shanghongcai@126.com; xingyanwei12345@163.com

${ }^{+}$Xinye Li, Xiandu Pan, Yanda Li and Na An contributed equally to this work.

${ }^{4}$ Key Laboratory of Chinese Internal Medicine of the Ministry of Education,

Dongzhimen Hospital Affiliated to Beijing University of Chinese Medicine,

Beijing, China

'Guang'anmen Hospital, China Academy of Chinese Medical Sciences,

Beijing, China

Full list of author information is available at the end of the article
}

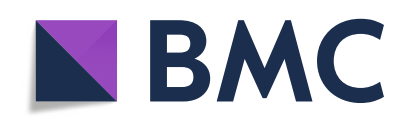

(c) The Author(s). 2020 Open Access This article is licensed under a Creative Commons Attribution 4.0 International License, which permits use, sharing, adaptation, distribution and reproduction in any medium or format, as long as you give appropriate credit to the original author(s) and the source, provide a link to the Creative Commons licence, and indicate if changes were made. The images or other third party material in this article are included in the article's Creative Commons licence, unless indicated otherwise in a credit line to the material. If material is not included in the article's Creative Commons licence and your intended use is not permitted by statutory regulation or exceeds the permitted use, you will need to obtain permission directly from the copyright holder. To view a copy of this licence, visit http://creativecommons.org/licenses/by/4.0/. The Creative Commons Public Domain Dedication waiver (http://creativecommons.org/publicdomain/zero/1.0/) applies to the data made available in this article, unless otherwise stated in a credit line to the data. 
(Continued from previous page)

Conclusions: COVID-19 patients with elevated Tnl levels are at significantly higher risk of severe disease, ICU admission, and death. Elevated CK, CK-MB, LDH, and IL-6 levels and emerging arrhythmia are associated with the development of severe disease and need for ICU admission, and the mortality is significantly higher in patients with elevated LDH and IL-6 levels.

Keywords: Cardiac injury, Biomarkers, COVID-19, Meta-analysis, Mortality

\section{Background}

Coronavirus disease (COVID-19) has spread worldwide, becoming a public health and medical care challenge in many countries. As of April 25, 2020, COVID-19 had spread to 213 countries, areas or territories, with 2,719, 897 confirmed cases and 187,705 confirmed deaths worldwide [1]. COVID-19, the clinical manifestation of severe acute respiratory syndrome coronavirus-2 (SARS-CoV-2) infection, is characterized by respiratory tract symptoms. Severe cases can involve acute respiratory distress syndrome (ARDS) and shock [2]. COVID-19 is considered mainly a respiratory tract disease, but cardiovascular complications can also occur, eventually leading to sudden deterioration [3, 4]. A large-scale study including 44,672 patients reported that cardiovascular disease was the risk factors for fatality of COVID-19 patients [5]. Intensive care unit (ICU) occupancy is very fluid, and COVID-19 patients still require better evidence-based cardiovascular treatment [6]. Inciardi et al. reported the case of a patient who recovered from the influenza-like syndrome but then developed symptoms of heart failure [3]. A recent study recommended that cardiac biomarkers should be evaluated in all hospitalized patients with confirmed COVID19 [7]. However, there has been less concern about cardiac complications in other published studies. Data such as those from transthoracic echocardiography, cardiac magnetic resonance imaging (MRI), coronary angiography, and other examinations of cardiovascular diseases, as well as the biomarkers of cardiac injury have been less often described or are even missing.

Recent case reports have suggested that acute cardiac injury can cause cardiac dysfunction, leading to cardiogenic shock and the proclivity for malignant arrhythmia [8]. Another study reported that COVID-19 was associated with myocarditis and arrhythmia [9]. Studies have shown that cardiac injury is related to higher in-hospital mortality rate [4] and is commonly observed in severe COVID-19 cases [9]. Therefore, paying attention to the occurrence of cardiac complications in patients with COVID-19 and performing risk stratification may greatly reduce patient mortality rates, especially of those with severe disease or requiring ICU admission. To our knowledge, this is the first study to comprehensively evaluate the impact of cardiac injury and its related biomarkers on mortality and other prognosis in patients infected with SARS-CoV-2.

\section{Methods}

Data sources and study selection

This meta-analysis was performed according to the Preferred Reporting Items for Systematic Reviews and Meta-analysis statement [10]. Two investigators (X.L. and Y.X.) independently conducted a comprehensive search of the relevant literature published until March 30, 2020, in the PubMed, EMBASE, Cochrane Library, MEDLINE, Chinese National Knowledge Infrastructure (CNKI), Wanfang, MedRxiv, and ChinaXiv databases. Combinations of the relevant medical subject heading $(\mathrm{MeSH})$ terms, key words, and word variants of "novel coronavirus," "coronavirus disease 2019," "COVID-19," "2019-nCoV," "SARS-2-CoV," "clinical or characteristic," and "relative risk or RR" were utilized to identify all potentially relevant studies. After the elimination of duplicates, the titles and abstracts of all retrieved studies were assessed by two independent reviewers (Y.L. and N.A.) to eliminate irrelevant articles. Any disagreements were settled by consensus or by a third reviewer. Language restrictions were not applied during filtering, to maximize search sensitivity.

The inclusion criteria were as follows: (1) diagnosis of COVID-19 according to the World Health Organization interim guidance [11] and (2) reported prognosis (severe disease, ICU admission, or death) with or without cardiac injury, reported cardiac injury biomarkers (for example, troponin $\mathrm{I}[\mathrm{TnI}]$, troponin $\mathrm{T}[\mathrm{TnT}]$, creatine kinase [CK], CK myocardial band [CK-MB], and lactate dehydrogenase $[\mathrm{LDH}])$, or arrhythmia. The exclusion criteria were as follows: (1) repeated articles, letters, editorials, and expert opinions and (2) studies with overlapping or unusable data. The primary outcome was the incidence of death, severe disease, or ICU admission in COVID-19 patients with elevated TnI levels versus nonelevated TnI levels. The secondary outcomes were as follows: (1) incidences of elevated TnI, CK, CK-MB, LDH, or interleukin-6 (IL-6) of the non-severe disease/nonICU versus severe disease/ICU groups; (2) incidences of elevated TnI, CK, CK-MB, LDH, or IL-6 of the survivors versus non-survivors groups; (3) TnI, CK, CK-MB, LDH, or IL-6 levels of the non-severe disease/non-ICU versus severe disease/ICU groups; (4) TnI, CK, CK-MB, LDH, or IL-6 levels of the survivors versus non-survivors groups; (5) incidence of arrhythmia (defined as newly 
occurring of any type) of the non-severe disease/nonICU versus severe disease/ICU groups.

\section{Data extraction}

Two investigators (X.L. and X.P.) independently extracted the relevant data from the eligible studies using predesigned forms. Disagreements were resolved by consensus. If the mean and standard deviation (SD) of the laboratory findings were not directly given, we used the estimation formula based on the median, range, and sample size [12]. Definitions used for severity assessment, ICU admission, and cardiac injury were also extracted.

\section{Quality assessment and publication bias}

Two researchers (X.P. and N.A.) independently assessed the quality of the included studies, using the NewcastleOttawa Quality Assessment Scale [13]. Studies were defined as high quality if a score of 7 or higher was attained [13]. Potential publication bias was evaluated using the visual inspection of funnel plots and formal testing with the Egger's testing [14].

\section{Statistical analysis}

Effect estimates are presented as relative risk (RR) or standard mean differences (SMD) with 95\% confidence interval (CI). The $I^{2}$ statistic was used to quantify the heterogeneity across studies. $I^{2}>50 \%$ suggested significant statistical heterogeneity [15]. In this case, a random-effects model was used considering the intraand interstudy variation. Otherwise, the pooled effect was calculated using a fixed-effects model. All analyses were performed using Stata 16.0 (StataCorp, College Station, TX, USA). Values of $P<0.05$ were considered statistically significant.

\section{Results}

Study selection

We identified 1898 studies using the predefined search terms. After the removal of duplicates and filtering of titles and abstracts to exclude irrelevant articles, 67 records remained. The full text of the 67 records was reviewed; of them, 44 records were excluded for the following reasons: data not available $(n=17)$, literature review or letter or case report $(n=12)$, unrelated to relevant predictive factors $(n=13)$, and meta-analysis

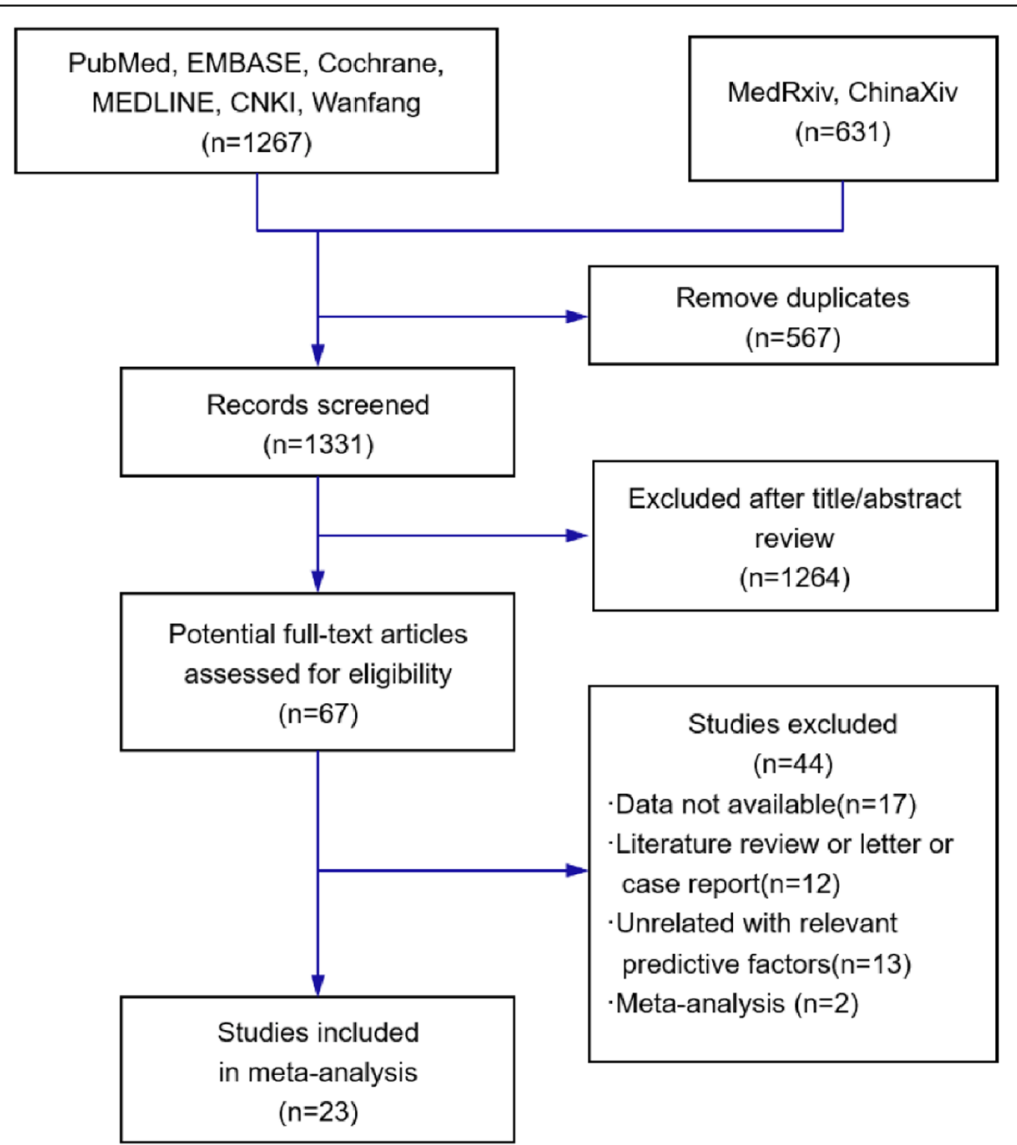

Fig. 1 Flow diagram showing study search and selection. CNKI=Chinese National Knowledge Infrastructure 
$(n=2)$. Finally, 23 studies were included in this metaanalysis, of which one was not written in English. The flow diagram of this study selection is shown in Fig. 1.

\section{Study characteristics}

The primary characteristics of the 23 included studies are listed in Table 1, with 4631 individuals incorporated. The sample size of 16 studies was greater than 100. The definition of cardiac injury was extracted (Table 2). The clinical characteristics of all included patients with COVID-19 are shown in Additional file 1: Table S1. Overall, 16 studies reported cardiac injury biomarkers, and 4 reported arrhythmias. All the results calculated using Stata are shown in Table 3.

\section{Non-elevated Tnl vs. elevated Tnl groups}

First, we restricted our analysis to studies that assessed the prognosis of COVID-19 patients with or without elevated TnI (or TnT). Nine studies reported the outcome events (including severe disease, ICU admission, and death) of patients with non-elevated or elevated TnI (TnT). TnI, which defines cardiac injury, was measured in a total of 1548 COVID-19 patients. The proportions of severe cases, ICU admissions, or death in the nonelevated $\mathrm{TnI}$ versus elevated $\mathrm{TnI}$ groups were $12.0 \%$ versus $64.5 \%, 11.8 \%$ versus $56.0 \%$, and $8.2 \%$ versus $59.3 \%$, respectively. Patients with elevated TnI levels had a significantly higher risk of severe disease, ICU admission, and death than those in the non-elevated TnI group (RR 5.57, $95 \%$ CI 3.04 to $10.22, P<0.001 ; I^{2}=78.6 \%$, Fig. 2a; RR 6.20, 95\% CI 2.52 to $15.29, P<0.001 ; I^{2}=89.3 \%$, Fig. 2b; RR 5.64, 95\% CI 2.69 to $11.83, P<0.001 ; I^{2}=$ $89.1 \%$, Fig. 2c, respectively). The mean $\mathrm{N}$-terminal proBNP (NT-proBNP) level was significantly higher in the elevated TnI group than in the non-elevated TnI group (SMD 1.63, 95\% CI 1.02 to 2.23, $P<0.001 ; I^{2}=86.6 \%$, Fig. 2d).

\section{Non-severe disease/non-ICU vs. severe disease/ICU groups and survivors vs. non-survivors groups Cardiac injury biomarkers}

Eight studies including 1028 patients in the non-severe disease/non-ICU group or severe disease/ICU group reported the number of patients with elevated TnI or TnT levels (total rate, 11.9\%). Elevated TnI or TnT levels occurred at a rate of $2.3 \%$ in the non-severe disease/nonICU group and $36.9 \%$ in the severe disease/ICU group. Patients in the severe/ICU group had an increased risk of developing elevated TnI or TnT levels (RR 15.10, 95\% CI 4.10 to $55.61, P<0.001 ; I^{2}=83.7 \%$, Fig. 3a). The mean TnI level was significantly higher in the severe disease/ICU admission group (SMD 0.74, 95\% CI 0.19 to $1.30, P=0.009 ; I^{2}=84.6 \%$, Fig. $\left.3 \mathrm{~b}\right)$. The proportion of patients with elevated $\mathrm{TnI}$ or TnT levels in the survivors and non-survivor groups was $14.3 \%$ and $63.9 \%$, respectively. Significantly more non-survivors than survivors had elevated TnI or TnT levels (RR 4.69, 95\% CI 3.39 to 6.48, $P<0.001 ; I^{2}=22.5 \%$, Fig. 3c). Twelve studies including 2174 individuals reported the CK levels or the number of patients with above-normal CK levels. The incidence of elevated $\mathrm{CK}$ in the severe disease/ICU group was significantly higher than that in the nonsevere disease/non-ICU group $(12.9 \%$ and $23.2 \%$, respectively; RR $1.98,95 \%$ CI 1.50 to $2.61, P<0.001 ; I^{2}=$ $0.0 \%$, Fig. 4a). The mean CK level was significantly higher in severe disease/ICU group than in the nonsevere disease/non-ICU group (SMD 0.39, 95\% CI 0.11 to $0.67, P=0.006 ; I^{2}=69.0 \%$, Fig. $\left.4 \mathrm{~b}\right)$. The proportion of patients with an elevated CK-MB level in the non-severe disease/non-ICU and severe disease/ICU groups was $14.1 \%$ and $45.7 \%$, respectively. Patients in the severe disease/ICU admission group were at higher risk of developing an elevated CK-MB level than those in the nonsevere disease/non-ICU group (RR 3.24, 95\% CI 1.66 to 6.34, $P=0.001 ; I^{2}=79.8 \%$, Fig. 4c). Of the 2532 patients from 15 studies, $29.7 \%$ of those in the non-severe disease/non-ICU group versus $60.1 \%$ of the severe disease/ ICU group had elevated LDH levels. COVID-19 patients with elevated LDH levels were at significantly increased risk of developing severe disease or requiring ICU admission (RR 2.20, 95\% CI 1.55 to $3.12, P<0.001 ; I^{2}=$ $79.7 \%$, Fig. 5a). LDH levels were significantly higher in the severe disease/ICU admission group than in the non-severe disease/non-ICU group (SMD 1.15, 95\% CI 0.61 to $1.70, P<0.001 ; I^{2}=92.7 \%$, Fig. $\left.5 \mathrm{~b}\right)$ and in nonsurvivors than in survivors (SMD 2.86, 95\% CI 0.67 to 5.06, $P=0.01 ; I^{2}=98.6 \%$, Fig. $5 \mathrm{c}$ ).

\section{Arrhythmia and IL-6}

The incidence of arrhythmia was $3.1 \%$ in the non-severe disease/non-ICU group versus $43.8 \%$ in the severe disease/ICU group. Patients with newly occurring arrhythmias were at a higher risk of developing severe disease or requiring ICU admission (RR 13.09, 95\% CI 7.00 to $24.47, P<0.001 ; I^{2}=42.0 \%$, Fig. $6 \mathrm{a}$ ). IL-6 levels were significantly higher in the severe disease/ICU group than in the non-severe disease/non-ICU group, as well as in non-survivors than in survivors (SMD 0.54, 95\% CI 0.27 to $0.81, P<0.001 ; I^{2}=0.0 \%$, Fig. 6 b; SMD $1.28,95 \% \mathrm{CI}$ 1.00 to $1.57, P<0.001 ; I^{2}=13.7 \%$, Fig. $6 c$, respectively).

\section{Discussion}

This systematic review and meta-analysis of 23 highquality retrospective studies systematically evaluated the risk of severe disease, ICU admission, or death associated with COVID-19-related cardiac injury performance. Our findings are as follows: (1) COVID-19 patients with elevated TnI levels are at significantly higher risk of 
Table 1 Characteristics of the studies included in the meta-analysis and systematic review

\begin{tabular}{|c|c|c|c|c|c|c|c|c|c|c|c|}
\hline Study & $\begin{array}{l}\text { Study period } \\
\text { and location }\end{array}$ & Study design & $\begin{array}{l}\text { Population, } \\
\boldsymbol{N}\end{array}$ & $\begin{array}{l}\text { Male, } \\
\boldsymbol{N}(\%)\end{array}$ & $\begin{array}{l}\text { Median/ } \\
\text { mean } \\
\text { age, year }\end{array}$ & $\begin{array}{l}\text { Non- } \\
\text { severe disease/ } \\
\text { severe disease, } \boldsymbol{N}\end{array}$ & $\begin{array}{l}\text { Non- } \\
\text { ICU/ } \\
\text { ICU, } \\
N\end{array}$ & $\begin{array}{l}\text { Survivors/ } \\
\text { Non- } \\
\text { survivors, } \\
\boldsymbol{N}\end{array}$ & $\begin{array}{l}\text { Definition } \\
\text { of severe } \\
\text { disease }\end{array}$ & $\begin{array}{l}\text { Study } \\
\text { population }\end{array}$ & $\begin{array}{l}\text { Quality } \\
\text { score }\end{array}$ \\
\hline $\begin{array}{l}\text { Wang } \\
\text { DW } \\
\text { et al. } \\
{[16]}\end{array}$ & $\begin{array}{l}\text { Jan } 1 \text { to Jan } \\
28,2020 \\
\text { Wuhan, } \\
\text { China }\end{array}$ & $\begin{array}{l}\text { SC, } \\
\text { retrospective } \\
\text { case series }\end{array}$ & 138 & $\begin{array}{l}75 \\
(54.3)\end{array}$ & $\begin{array}{l}56 \text { (IQR: } \\
42-68)\end{array}$ & NA & $\begin{array}{l}102 / \\
36\end{array}$ & NA & $\begin{array}{l}\text { Developed } \\
\text { ARDS }\end{array}$ & $\begin{array}{l}\text { Consecutive } \\
\text { hospitalized } \\
\text { patients } \\
\text { with } \\
\text { confirmed } \\
\text { NCIP }\end{array}$ & 9 \\
\hline $\begin{array}{l}\text { Wu CM } \\
\text { et al. } \\
\text { (a) [17] }\end{array}$ & $\begin{array}{l}\text { Dec 25, } \\
\text { 2019, to Jan } \\
\text { 26, 2020, } \\
\text { Wuhan, } \\
\text { China }\end{array}$ & $\begin{array}{l}\text { SC, } \\
\text { retrospective } \\
\text { cohort study }\end{array}$ & $201^{*}$ & $\begin{array}{l}128 \\
(63.7)\end{array}$ & $\begin{array}{l}51 \text { (IQR: } \\
43-60)\end{array}$ & $117 / 84$ & NA & $40 / 44$ & $\begin{array}{l}\text { Developed } \\
\text { ARDS }\end{array}$ & $\begin{array}{l}\text { Patients } \\
\text { with } \\
\text { confirmed } \\
\text { COVID-19 } \\
\text { pneumonia }\end{array}$ & 9 \\
\hline $\begin{array}{l}\text { Yang } \\
\text { XB } \\
\text { et al. } \\
{[18]}\end{array}$ & $\begin{array}{l}\text { Dec 24, } \\
\text { 2019, to Jan } \\
\text { 26, 2020, } \\
\text { Wuhan, } \\
\text { China }\end{array}$ & $\begin{array}{l}\text { SC, } \\
\text { retrospective, } \\
\text { observational } \\
\text { study }\end{array}$ & 52 & $\begin{array}{l}35 \\
(67.3)\end{array}$ & $\begin{array}{l}59.7 \text { (SD: } \\
13.3)\end{array}$ & NA & NA & 20/32 & NA & $\begin{array}{l}\text { Critically ill } \\
\text { patients } \\
\text { with SARS- } \\
\text { CoV-2 } \\
\text { pneumonia }\end{array}$ & 8 \\
\hline $\begin{array}{l}\text { Huang } \\
C L \\
\text { et al. } \\
{[2]}\end{array}$ & $\begin{array}{l}\text { Dec 16, } \\
\text { 2019, to Jan } \\
\text { 2, 2020, } \\
\text { Wuhan, } \\
\text { China }\end{array}$ & $\begin{array}{l}N A \text {, } \\
\text { retrospective }\end{array}$ & 41 & $\begin{array}{l}30 \\
(73.2)\end{array}$ & $\begin{array}{l}49 \text { (IQR: } \\
41-58)\end{array}$ & NA & $\begin{array}{l}28 / \\
13\end{array}$ & $35 / 6$ & $\begin{array}{l}\text { Required high- } \\
\text { flow nasal can- } \\
\text { nula or higher- } \\
\text { level oxygen } \\
\text { support mea- } \\
\text { sures to correct } \\
\text { hypoxemia }\end{array}$ & $\begin{array}{l}\text { Patients } \\
\text { identified as } \\
\text { having } \\
\text { laboratory- } \\
\text { confirmed } \\
2019-n C o V \\
\text { infection } \\
\text { and admit- } \\
\text { ted hospital }\end{array}$ & 8 \\
\hline $\begin{array}{l}\text { Chen } \\
\text { D et al. } \\
\text { [19] }\end{array}$ & $\begin{array}{l}\text { Jan } 11 \text { to } \\
\text { Feb } 15, \\
\text { 2020, } \\
\text { Wenzhou, } \\
\text { China, }\end{array}$ & $\begin{array}{l}\text { MC, } \\
\text { retrospective } \\
\text { study }\end{array}$ & 175 & $\begin{array}{l}83 \\
(47.4)\end{array}$ & $\begin{array}{l}46 \text { (IQR: } \\
34-54)\end{array}$ & $40 / 135$ & NA & NA & $\begin{array}{l}\text { Showed } \\
\text { pneumonia and } \\
\text { any of acute } \\
\text { respiratory } \\
\text { distress } \\
\text { syndrome }\end{array}$ & $\begin{array}{l}\text { Patients } \\
\text { with COVID- } \\
19\end{array}$ & 8 \\
\hline $\begin{array}{l}\text { Fu L } \\
\text { et al. } \\
\text { [20] }\end{array}$ & $\begin{array}{l}\text { Jan } 1 \text { to Jan } \\
30,2020, \\
\text { Wuhan, } \\
\text { China }\end{array}$ & $\begin{array}{l}\text { SC, } \\
\text { retrospective } \\
\text { cohort study }\end{array}$ & 200 & $\begin{array}{l}99 \\
(49.5)\end{array}$ & NA & NA & NA & $166 / 34$ & NA & $\begin{array}{l}\text { Patients } \\
\text { with } \\
\text { confirmed } \\
\text { COVID-19 }\end{array}$ & 8 \\
\hline $\begin{array}{l}\text { Guan } \\
\text { WJ } \\
\text { et al. } \\
{[21]}\end{array}$ & $\begin{array}{l}\text { Jan } 1 \text { to Jan } \\
29,2020 \text {, } \\
\text { China }\end{array}$ & $\begin{array}{l}\text { MC, } \\
\text { retrospective } \\
\text { study }\end{array}$ & 1099 & $\begin{array}{l}640 \\
(58.2)\end{array}$ & $\begin{array}{l}47 \text { (IQR: } \\
35-58)\end{array}$ & $926 / 173$ & NA & $1084 / 15$ & NA & $\begin{array}{l}\text { Patients } \\
\text { with } \\
\text { laboratory- } \\
\text { confirmed } \\
\text { 2019-nCoV } \\
\text { ARD }\end{array}$ & 8 \\
\hline $\begin{array}{l}\text { Hui H } \\
\text { et al. } \\
{[22]}\end{array}$ & $\begin{array}{l}\text { Jan } 21 \text { to } \\
\text { Feb 03, } \\
\text { 2020, Beijing, } \\
\text { China }\end{array}$ & $\begin{array}{l}\text { SC, } \\
\text { retrospective } \\
\text { study }\end{array}$ & 41 & $\begin{array}{l}19 \\
(46.3)\end{array}$ & $\begin{array}{l}47 \text { (IQR: } \\
35.5-64)\end{array}$ & $34 / 7$ & NA & NA & NA & $\begin{array}{l}\text { Patients } \\
\text { with } \\
\text { confirmed } \\
\text { COVID-19 }\end{array}$ & 7 \\
\hline $\begin{array}{l}\text { Liu YL } \\
\text { et al. } \\
\text { [23] }\end{array}$ & $\begin{array}{l}\text { Jan } 2 \text { to Feb } \\
12,2020, \\
\text { Wuhan, } \\
\text { China }\end{array}$ & $\begin{array}{l}\mathrm{SC} \\
\text { retrospective } \\
\text { study }\end{array}$ & 109 & $\begin{array}{l}59 \\
(54.1)\end{array}$ & $\begin{array}{l}55 \text { (IQR: } \\
43-66)\end{array}$ & $56 / 53$ & NA & $78 / 31$ & $\begin{array}{l}\text { Developed } \\
\text { ARDS }\end{array}$ & $\begin{array}{l}\text { Patients } \\
\text { with } \\
\text { confirmed } \\
\text { CovID-19 }\end{array}$ & 8 \\
\hline $\begin{array}{l}\text { Liu L } \\
\text { et al. } \\
{[24]}\end{array}$ & $\begin{array}{l}\text { Jan } 20 \text { to } \\
\text { Feb 3, 2020, } \\
\text { Chongqing, } \\
\text { China }\end{array}$ & $\begin{array}{l}\text { SC, } \\
\text { retrospective } \\
\text { case series }\end{array}$ & 51 & $\begin{array}{l}32 \\
(62.7)\end{array}$ & $\begin{array}{l}45 \text { (IQR: } \\
34-51)\end{array}$ & $44 / 7$ & NA & NA & NA & $\begin{array}{l}\text { Patients } \\
\text { with } \\
\text { confirmed } \\
\text { covID-19 }\end{array}$ & 8 \\
\hline $\begin{array}{l}\text { Qi D } \\
\text { et al. } \\
\text { [25] }\end{array}$ & $\begin{array}{l}\text { Jan } 19 \text { to } \\
\text { Feb 16, } \\
\text { 2020, } \\
\text { Chongqing, } \\
\text { China }\end{array}$ & $\begin{array}{l}\text { MC, } \\
\text { retrospective, } \\
\text { descriptive } \\
\text { study }\end{array}$ & 267 & $\begin{array}{l}149 \\
(55.8)\end{array}$ & $\begin{array}{l}48 \text { (IQR: } \\
35-65)\end{array}$ & $217 / 50$ & $\begin{array}{l}214 / \\
53\end{array}$ & $263 / 4$ & $\begin{array}{l}\text { According to } \\
\text { the American } \\
\text { Thoracic } \\
\text { Society } \\
\text { guideline }\end{array}$ & $\begin{array}{l}\text { Patients } \\
\text { with COVID- } \\
19 \text { con- } \\
\text { firmed by } \\
\text { real-time RT- } \\
\text { PCR }\end{array}$ & 7 \\
\hline
\end{tabular}


Table 1 Characteristics of the studies included in the meta-analysis and systematic review (Continued)

\begin{tabular}{|c|c|c|c|c|c|c|c|c|c|c|c|}
\hline Study & $\begin{array}{l}\text { Study period } \\
\text { and location }\end{array}$ & Study design & $\begin{array}{l}\text { Population, } \\
\boldsymbol{N}\end{array}$ & $\begin{array}{l}\text { Male, } \\
\boldsymbol{N}(\%)\end{array}$ & $\begin{array}{l}\text { Median/ } \\
\text { mean } \\
\text { age, year }\end{array}$ & $\begin{array}{l}\text { Non- } \\
\text { severe disease/ } \\
\text { severe disease, } \boldsymbol{N}\end{array}$ & $\begin{array}{l}\text { Non- } \\
\text { ICU/ } \\
\text { ICU, } \\
N\end{array}$ & $\begin{array}{l}\text { Survivors/ } \\
\text { Non- } \\
\text { survivors, } \\
N\end{array}$ & $\begin{array}{l}\text { Definition } \\
\text { of severe } \\
\text { disease }\end{array}$ & $\begin{array}{l}\text { Study } \\
\text { population }\end{array}$ & $\begin{array}{l}\text { Quality } \\
\text { score }\end{array}$ \\
\hline $\begin{array}{l}\text { Wang } \\
\text { YF } \\
\text { et al. } \\
{[26]}\end{array}$ & $\begin{array}{l}\text { Jan } 1 \text { to Feb } \\
\text { 10, 2020, } \\
\text { Wuhan, } \\
\text { China }\end{array}$ & $\begin{array}{l}\mathrm{SC}, \\
\text { retrospective }\end{array}$ & 110 & $\begin{array}{l}48 \\
(43.6)\end{array}$ & NA & $72 / 38$ & NA & $\mathrm{NA}$ & $\begin{array}{l}\text { Fever or } \\
\text { suspected } \\
\text { respiratory } \\
\text { infection, plus } \\
\text { one of a } \\
\text { respiratory } \\
\text { rate }>30 \\
\text { breaths/min, } \\
\text { severe } \\
\text { respiratory } \\
\text { distress, or } \\
\text { SpO2 }<90 \% \text { on } \\
\text { room air }\end{array}$ & $\begin{array}{l}\text { Patients } \\
\text { with } \\
\text { confirmed } \\
\text { COVID-19 } \\
\text { pneumonia }\end{array}$ & 8 \\
\hline $\begin{array}{l}\text { Wu CM } \\
\text { et al. } \\
\text { (b) [27] }\end{array}$ & $\begin{array}{l}\text { Dec 25, } 2019 \\
\text { to Jan 27, } \\
\text { 2020, } \\
\text { Wuhan, } \\
\text { China }\end{array}$ & $\begin{array}{l}\text { SC, } \\
\text { retrospective } \\
\text { cohort study }\end{array}$ & 188 & $\begin{array}{l}119 \\
(63.3)\end{array}$ & $\begin{array}{l}51.9 \text { (SD: } \\
14.26)\end{array}$ & NA & $\begin{array}{l}138 / \\
50\end{array}$ & $145 / 4$ & NA & $\begin{array}{l}\text { Patients } \\
\text { with } \\
\text { confirmed } \\
\text { COVID-19 } \\
\text { pneumonia }\end{array}$ & 8 \\
\hline $\begin{array}{l}\text { Xu HY } \\
\text { et al. } \\
\text { [28] }\end{array}$ & $\begin{array}{l}\text { Jan } 02 \text { to } \\
\text { Feb 14, } \\
2020, \text { NA }\end{array}$ & $\begin{array}{l}\mathrm{NA} \text {, } \\
\text { retrospective }\end{array}$ & 53 & $\begin{array}{l}28 \\
(52.8)\end{array}$ & NA & $34 / 19$ & $45 / 8$ & $53 / 3$ & $\begin{array}{l}\text { More likely to } \\
\text { have } \\
\text { underlying } \\
\text { comorbidities, } \\
\text { and AMI }\end{array}$ & $\begin{array}{l}\text { Consecutive } \\
\text { laboratory- } \\
\text { confirmed } \\
\text { and hospi- } \\
\text { talized pa- } \\
\text { tients with } \\
\text { confirmed } \\
\text { NCIP }\end{array}$ & 7 \\
\hline $\begin{array}{l}\text { Xu YH } \\
\text { et al. } \\
\text { [29] }\end{array}$ & $\begin{array}{l}\text { Jan } 14 \text { to } \\
\text { Feb 28, } \\
2020, \\
\text { Guangdong, } \\
\text { China }\end{array}$ & $\begin{array}{l}\text { MC, } \\
\text { retrospective, } \\
\text { observational } \\
\text { study }\end{array}$ & 45 & $\begin{array}{l}29 \\
(64.4)\end{array}$ & $\begin{array}{l}56.7 \text { (SD: } \\
15.4)\end{array}$ & $25 / 20$ & $0 / 45$ & $44 / 1$ & $\begin{array}{l}\text { Defined as } \\
\text { those required } \\
\text { oxygen therapy, } \\
\text { symptoms of } \\
\text { respiratory } \\
\text { distress or } \\
\text { required } \\
\text { mechanical } \\
\text { ventilation }\end{array}$ & $\begin{array}{l}\text { critically ill } \\
\text { patients } \\
\text { with SARS- } \\
\text { CoV-2 } \\
\text { pneumonia } \\
\text { RT-PCR } \\
\text { confirmed } \\
\text { positive } \\
\text { patients }\end{array}$ & 8 \\
\hline $\begin{array}{l}\text { Liu YB } \\
\text { et al. } \\
\text { [30] }\end{array}$ & $\begin{array}{l}\text { Jan } 10 \text { to } \\
\text { Feb 24, } \\
\text { 2020, } \\
\text { Guangzhou, } \\
\text { China }\end{array}$ & $\begin{array}{l}\text { SC, } \\
\text { retrospective }\end{array}$ & 291 & $\begin{array}{l}133 \\
(45.7)\end{array}$ & $\begin{array}{l}48.1 \\
\text { (IQR: } 34- \\
62)\end{array}$ & $262 / 29$ & $\begin{array}{l}265 / \\
26\end{array}$ & $290 / 1$ & NA & $\begin{array}{l}\text { Laboratory- } \\
\text { confirmed } \\
\text { patients } \\
\text { with NCIP }\end{array}$ & 8 \\
\hline $\begin{array}{l}\text { Peng } \\
\text { YD } \\
\text { et al. } \\
{[31]}\end{array}$ & $\begin{array}{l}\text { Jan } 20 \text { to } \\
\text { Feb, 15, } \\
2020, \\
\text { Wuhan, } \\
\text { China }\end{array}$ & $\begin{array}{l}\text { SC, } \\
\text { retrospective, } \\
\text { cohort study }\end{array}$ & 112 & $\begin{array}{l}53 \\
(47.0)\end{array}$ & $\begin{array}{l}62 \text { (IQR: } \\
55-67)\end{array}$ & $96 / 16$ & NA & $84 / 28$ & $\begin{array}{l}\text { Required } \\
\text { mechanical } \\
\text { ventilation; } \\
\text { shock; } \\
\text { combined with } \\
\text { other organ } \\
\text { failure }\end{array}$ & $\begin{array}{l}\text { COVID-19 } \\
\text { patients } \\
\text { with CVD }\end{array}$ & 7 \\
\hline $\begin{array}{l}\text { Zhang } \\
\text { GQ } \\
\text { et al. } \\
\text { [32] }\end{array}$ & $\begin{array}{l}\text { Jan } 2 \text { to Feb, } \\
10,2020, \\
\text { Wuhan, } \\
\text { China }\end{array}$ & $\begin{array}{l}\text { SC, } \\
\text { retrospective } \\
\text { case series } \\
\text { study }\end{array}$ & 221 & $\begin{array}{l}108 \\
(48.9)\end{array}$ & $\begin{array}{l}55 \text { (IQR: } \\
\text { 39-66.5) }\end{array}$ & $166 / 55$ & NA & 209/12 & $\begin{array}{l}\text { Fever plus one } \\
\text { of these } \\
\text { conditions, } \\
\text { including } \\
\text { respiratory } \\
\text { rate } \geq 30 \\
\text { breaths/min, } \\
\text { severe } \\
\text { respiratory } \\
\text { distress, } \\
\text { SpO2 } \leq 93 \% \text { on } \\
\text { room air, } \\
\text { occurrence of } \\
\text { respiratory } \\
\text { failure requiring }\end{array}$ & $\begin{array}{l}\text { Patients } \\
\text { who were } \\
\text { confirmed } \\
\text { diagnosed } \\
\text { as COVID-19 } \\
\text { according to } \\
\text { WHO in- } \\
\text { terim } \\
\text { guidance }\end{array}$ & 8 \\
\hline
\end{tabular}


Table 1 Characteristics of the studies included in the meta-analysis and systematic review (Continued)

\begin{tabular}{|c|c|c|c|c|c|c|c|c|c|c|c|}
\hline Study & $\begin{array}{l}\text { Study period } \\
\text { and location }\end{array}$ & Study design & $\begin{array}{l}\text { Population, } \\
\boldsymbol{N}\end{array}$ & $\begin{array}{l}\text { Male, } \\
\boldsymbol{N}(\%)\end{array}$ & $\begin{array}{l}\text { Median/ } \\
\text { mean } \\
\text { age, year }\end{array}$ & $\begin{array}{l}\text { Non- } \\
\text { severe disease/ } \\
\text { severe disease, } \boldsymbol{N}\end{array}$ & $\begin{array}{l}\text { Non- } \\
\text { ICU/ } \\
\text { ICU, } \\
N\end{array}$ & $\begin{array}{l}\text { Survivors/ } \\
\text { Non- } \\
\text { survivors, } \\
\boldsymbol{N}\end{array}$ & $\begin{array}{l}\text { Definition } \\
\text { of severe } \\
\text { disease }\end{array}$ & $\begin{array}{l}\text { Study } \\
\text { population }\end{array}$ & $\begin{array}{l}\text { Quality } \\
\text { score }\end{array}$ \\
\hline & & & & & & & & & $\begin{array}{l}\text { mechanical } \\
\text { ventilation, } \\
\text { shock and } \\
\text { other organ } \\
\text { failure }\end{array}$ & & \\
\hline $\begin{array}{l}\text { Liu T } \\
\text { et al. } \\
\text { [33] }\end{array}$ & $\begin{array}{l}\text { Jan 21, to } \\
\text { Feb 16, } \\
2020, \\
\text { Wuhan, } \\
\text { China }\end{array}$ & $\begin{array}{l}\mathrm{NA} \text {, } \\
\text { retrospective }\end{array}$ & 80 & $\begin{array}{l}34 \\
(42.5)\end{array}$ & $\begin{array}{l}53 \\
\text { (range: } \\
\text { 26-86) }\end{array}$ & $11 / 69$ & NA & $80 / 0$ & $\begin{array}{l}\text { Defined when } \\
\text { any of the } \\
\text { following } \\
\text { criteria was } \\
\text { met: dyspnea, } \\
\text { respiration } \\
\text { rate } \geq 30 \text { times/ } \\
\text { min; oxygen } \\
\text { saturation by } \\
\text { pulse oximeter } \\
\leq 93 \% \text { in resting } \\
\text { state; partial } \\
\text { pressure of } \\
\text { arterial oxygen } \\
\text { to fraction of } \\
\text { inspired oxygen } \\
\text { ratio } \leq 300 \\
\text { mmHg }\end{array}$ & $\begin{array}{l}\text { SARS-CoV-2 } \\
\text { nucleic acid } \\
\text { or RT-PCR } \\
\text { confirmed } \\
\text { positive } \\
\text { patients }\end{array}$ & 8 \\
\hline $\begin{array}{l}\text { Shi SB } \\
\text { et al. } \\
{[4]}\end{array}$ & $\begin{array}{l}\text { Jan 20, to } \\
\text { Feb 10, } \\
2020, \\
\text { Wuhan, } \\
\text { China }\end{array}$ & $\begin{array}{l}\text { SC, } \\
\text { retrospective } \\
\text { cohort study }\end{array}$ & 416 & $\begin{array}{l}205 \\
(49.3)\end{array}$ & $\begin{array}{l}64 \\
\text { (range: } \\
\text { 21-95) }\end{array}$ & $319 / 97$ & NA & $359 / 57$ & NA & $\begin{array}{l}\text { Consecutive } \\
\text { inpatients } \\
\text { with } \\
\text { laboratory- } \\
\text { confirmed } \\
\text { COVID-19 }\end{array}$ & 9 \\
\hline $\begin{array}{l}\text { Wu J } \\
\text { et al. } \\
\text { [34] }\end{array}$ & $\begin{array}{l}\text { Jan } 20 \text { to } \\
\text { Feb 20,2020, } \\
\text { Jiangsu and } \\
\text { Anhui } \\
\text { Province, } \\
\text { China }\end{array}$ & $\begin{array}{l}\text { MC, } \\
\text { retrospective } \\
\text { case series }\end{array}$ & 280 & $\begin{array}{l}151 \\
(53.9)\end{array}$ & $\begin{array}{l}43.1 \text { (SD: } \\
19.02)\end{array}$ & $197 / 83$ & NA & NA & NA & $\begin{array}{l}\text { Patients } \\
\text { infected } \\
\text { with SARS- } \\
\text { CoV-2 }\end{array}$ & 8 \\
\hline $\begin{array}{l}\text { Chen T } \\
\text { et al. } \\
\text { [35] }\end{array}$ & $\begin{array}{l}\text { Jan } 13 \text {, to } \\
\text { Feb 28, } \\
2020, \\
\text { Wuhan, } \\
\text { China }\end{array}$ & $\begin{array}{l}\text { SC, } \\
\text { retrospective }\end{array}$ & 274 & $\begin{array}{l}171 \\
(62)\end{array}$ & $\begin{array}{l}62 \text { (IQR: } \\
44-70)\end{array}$ & NA & NA & $161 / 113$ & NA & $\begin{array}{l}\text { Patients } \\
\text { with } \\
\text { confirmed } \\
\text { COVID-19 } \\
\text { pneumonia }\end{array}$ & 8 \\
\hline $\begin{array}{l}\text { Guo T } \\
\text { et al. } \\
\text { [36] }\end{array}$ & $\begin{array}{l}\text { Jan 23, to } \\
\text { Feb 23, } \\
\text { 2020, } \\
\text { Wuhan, } \\
\text { China }\end{array}$ & $\begin{array}{l}\text { SC, } \\
\text { retrospective }\end{array}$ & 187 & $\begin{array}{l}91 \\
(48.7)\end{array}$ & $\begin{array}{l}58.5 \text { (SD: } \\
14.66)\end{array}$ & NA & NA & $144 / 43$ & NA & $\begin{array}{l}\text { Patients } \\
\text { with } \\
\text { confirmed } \\
\text { COVID-19 } \\
\text { pneumonia }\end{array}$ & 9 \\
\hline
\end{tabular}

$A M I$ acute myocardial injury, ARDS acute respiratory distress syndrome, COVID-19 coronavirus disease 2019, CVD cardiovascular disease, 2019-nCoV novel coronavirus, $I Q R$ interquartile range, $M C$ multicenter study, $N$ number, $N A$ not available, $N C I P$ novel coronavirus-infected pneumonia, $R T-P C R$ reverse transcriptase polymerase chain reaction, SARS-CoV-2 severe acute respiratory syndrome coronavirus-2, SC single-center study, SD standard deviation

developing severe disease, requiring ICU admission, or death; (2) elevated CK, CK-MB, LDH, and IL-6 levels and emerging arrhythmia are associated with the development of severe disease or requirement for ICU admission; and (3) mortality rates are significantly higher among patients with elevated LDH and IL-6 levels.

\section{Cardiac injury}

Cardiac injury was defined as a serum cardiac biomarker level (e.g., troponin I) above the 99th percentile upper reference limit or new abnormalities seen on electrocardiography (ECG) and echocardiography [2]. CK, CK$\mathrm{MB}$, and $\mathrm{LDH}$ are also indicators associated with cardiac injury [37, 38]. An elevated cardiac TnI level has high specificity for cardiac injury and is a preferred biomarker of cardiac injury. Overall, in 8 studies including 1028 patients, the rates of elevated TnI or TnT in the nonsevere disease/non-ICU admission group and severe disease/ICU admission group were $2.3 \%$ and $36.9 \%$, respectively; in the total population, elevated TnI or TnT 
Table 2 Clinical characteristics of COVID-19 patients with or without elevated Tnl (TnT)

\begin{tabular}{|c|c|c|c|c|c|c|c|c|c|}
\hline \multirow[t]{2}{*}{ Source } & \multicolumn{2}{|c|}{ Severe disease/ARDS } & \multicolumn{2}{|l|}{$\mathrm{ICU}$} & \multicolumn{2}{|l|}{ Death } & \multicolumn{2}{|c|}{$\begin{array}{l}\text { NT-proBNP, Median } \\
(\mathrm{IQR}), \mathrm{pg} / \mathrm{mL}\end{array}$} & \multirow{2}{*}{$\begin{array}{l}\text { Definition } \\
\text { of cardiac } \\
\text { injury }\end{array}$} \\
\hline & $\begin{array}{l}\text { Elevated Tnl/ } \\
\text { TnT, } \boldsymbol{N} / \text { total }\end{array}$ & $\begin{array}{l}\text { Non-elevated } \\
\text { Tnl/TnT, } \boldsymbol{N} / \text { total }\end{array}$ & $\begin{array}{l}\text { Elevated Tnl/ } \\
\text { TnT, } \boldsymbol{N} / \text { total }\end{array}$ & $\begin{array}{l}\text { Non-elevated } \\
\text { Tnl/TnT, } \boldsymbol{N} / \text { total }\end{array}$ & $\begin{array}{l}\text { Elevated Tnl/ } \\
\text { TnT, } \boldsymbol{N} / \text { total }\end{array}$ & $\begin{array}{l}\text { Non-elevated } \\
\text { Tnl/TnT, } \boldsymbol{N} / \text { total }\end{array}$ & $\begin{array}{l}\text { Elevated } \\
\text { Tnl/TnT }\end{array}$ & $\begin{array}{l}\text { Non- } \\
\text { elevated } \\
\text { Tnl/TnT }\end{array}$ & \\
\hline $\begin{array}{l}\text { Liu et al. } \\
\text { [30] }\end{array}$ & $11 / 15$ & $18 / 276$ & $11 / 15$ & $15 / 276$ & $1 / 15$ & $0 / 276$ & NA & NA & $\begin{array}{l}\text { Tnl > } \\
0.03 \mu \mathrm{g} / \mathrm{L}\end{array}$ \\
\hline $\begin{array}{l}\text { Xu et al. } \\
\text { [28] }\end{array}$ & $6 / 6$ & $13 / 47$ & $6 / 6$ & $2 / 47$ & $3 / 6$ & $0 / 47$ & NA & NA & $\begin{array}{l}\text { TnT-HSST } \\
>28 \mathrm{pg} / \mathrm{ml}\end{array}$ \\
\hline $\begin{array}{l}\text { Wu et al. } \\
\text { (b) [27] }\end{array}$ & NA & NA & $27 / 62$ & $23 / 126$ & $31 / 62$ & $12 / 126$ & NA & NA & $\begin{array}{l}\text { Hs-Tnl } \geq \\
6.126 \mathrm{pg} / \\
\mathrm{mL}\end{array}$ \\
\hline $\begin{array}{l}\text { Hui et al. } \\
\text { [22] }\end{array}$ & $4 / 4$ & $1 / 16$ & NA & NA & NA & NA & NA & NA & NA \\
\hline $\begin{array}{l}\text { Wang } \\
\text { et al. } \\
{[16]}\end{array}$ & NA & NA & $8 / 10$ & $28 / 128$ & NA & NA & NA & NA & NA \\
\hline $\begin{array}{l}\text { Yang } \\
\text { et al. } \\
{[18]}\end{array}$ & NA & NA & NA & NA & $9 / 12$ & $23 / 40$ & NA & NA & $\begin{array}{l}\mathrm{Hs}-\mathrm{TNI}> \\
28 \mathrm{pg} / \mathrm{mL}\end{array}$ \\
\hline $\begin{array}{l}\text { Shi et al. } \\
{[4]}\end{array}$ & $48 / 82$ & $49 / 334$ & NA & NA & $42 / 82$ & $15 / 334$ & $\begin{array}{l}1689(698- \\
3327)\end{array}$ & $\begin{array}{l}139(51- \\
335)\end{array}$ & $\begin{array}{l}\mathrm{Hs}-\mathrm{Tnl}> \\
0.04 \mathrm{ng} / \mathrm{mL}\end{array}$ \\
\hline $\begin{array}{l}\text { Chen } \\
\text { et al. } \\
\text { [35] }\end{array}$ & NA & NA & NA & NA & $68 / 83$ & $26 / 120$ & NA & NA & $\begin{array}{l}\mathrm{Hs}-\mathrm{Tnl}> \\
15.6 \mathrm{pg} / \mathrm{mL}\end{array}$ \\
\hline $\begin{array}{l}\text { Guo } \\
\text { et al. } \\
\text { [36] }\end{array}$ & NA & NA & NA & NA & $31 / 52$ & $12 / 135$ & $\begin{array}{l}817.4 \\
(336.0- \\
1944.0)\end{array}$ & $\begin{array}{l}141.4(39.3- \\
303.6)\end{array}$ & $\begin{array}{l}\text { Elevated } \\
\text { TnT levels }\end{array}$ \\
\hline
\end{tabular}

ARDS acute respiratory distress syndrome, $H s$-Tnl high-sensitivity troponin I, ICU intensive care unit, IQR interquartile range, $N$ number, NA not available, NT-proBNP $\mathrm{N}$-terminal pro-B-type natriuretic peptide, $T n l$ troponin I, $T n T$ troponin T, TnT-HSST troponin T-hypersensitivity

Table 3 All the results calculated using Stata

\begin{tabular}{|c|c|c|c|c|c|c|c|c|c|c|c|c|c|c|c|}
\hline \multirow[t]{2}{*}{ Characteristic } & \multicolumn{5}{|c|}{$\begin{array}{l}\text { Non-elevated Tnl/TnT vs. elevated Tnl/ } \\
\text { TnT }\end{array}$} & \multicolumn{5}{|c|}{$\begin{array}{l}\text { Non-severe disease/non-ICU vs. severe } \\
\text { disease/ICU }\end{array}$} & \multicolumn{5}{|c|}{ Survivors vs. non-survivors } \\
\hline & $\overline{I^{2}(\%)}$ & $\mathrm{RR}$ & $95 \% \mathrm{Cl}$ & $Z$ & $P$ & $\overline{I^{2}(\%)}$ & $\mathrm{RR} / \mathrm{SMD}$ & $95 \% \mathrm{Cl}$ & $Z$ & $P$ & $I^{2}(\%)$ & RR/SMD & $95 \% \mathrm{Cl}$ & $Z$ & $P$ \\
\hline Severe, $n$ & 78.6 & 5.57 & $(3.04,10.22)$ & 5.55 & 0.00 & NA & NA & NA & NA & NA & NA & NA & NA & NA & NA \\
\hline$I C U, n$ & 89.3 & 6.20 & $(2.52,15.29)$ & 3.96 & 0.00 & NA & NA & NA & NA & NA & NA & NA & NA & NA & NA \\
\hline Death, $n$ & 89.1 & 5.64 & $(2.69,11.83)$ & 4.57 & 0.00 & NA & NA & NA & NA & NA & NA & NA & NA & NA & NA \\
\hline NT-proBNP (pg/ml) & 86.6 & 1.63 & $(1.02,2.23)$ & 5.27 & 0.00 & NA & NA & NA & NA & NA & NA & NA & NA & NA & NA \\
\hline Elevated Tnl/TnT, $n$ & NA & NA & NA & NA & NA & 83.7 & 15.10 & $(4.10,55.61)$ & 4.08 & 0.00 & 22.5 & 4.69 & $(3.39,6.48)$ & 9.37 & 0.00 \\
\hline Tnl (pg/ml) & NA & NA & NA & NA & NA & 84.6 & 0.74 & $(0.19,1.30)$ & 2.62 & 0.009 & NA & NA & NA & NA & NA \\
\hline Elevated CK, $n$ & NA & NA & NA & NA & NA & 0.0 & 1.98 & $(1.50,2.61)$ & 4.78 & 0.00 & NA & NA & NA & NA & NA \\
\hline CK (U/L) & NA & NA & NA & NA & NA & 69.0 & 0.39 & $(0.11,0.67)$ & 2.76 & 0.006 & NA & NA & NA & NA & NA \\
\hline Elevated CK-MB, $n$ & NA & NA & NA & NA & NA & 79.8 & 3.24 & $(1.66,6.34)$ & 3.44 & 0.001 & NA & NA & NA & NA & NA \\
\hline CK-MB (U/L) & NA & NA & NA & NA & NA & NA & NA & NA & NA & NA & NA & NA & NA & NA & NA \\
\hline Elevated LDH, $n$ & NA & NA & NA & NA & NA & 79.7 & 2.20 & $(1.55,3.12)$ & 4.40 & 0.00 & NA & NA & NA & NA & NA \\
\hline LDH (U/L) & NA & NA & NA & NA & NA & 92.7 & 1.15 & $(0.61,1.70)$ & 4.16 & 0.00 & 98.6 & 2.86 & $(0.67,5.06)$ & 2.56 & 0.01 \\
\hline BNP (pg/ml) & NA & NA & NA & NA & NA & NA & NA & NA & NA & NA & NA & NA & NA & NA & NA \\
\hline Arrhythmia, $n$ & NA & NA & NA & NA & NA & 42.0 & 13.09 & $(7.00,24.47)$ & 8.06 & 0.00 & NA & NA & NA & NA & NA \\
\hline IL-6 (pg/ml) & NA & NA & NA & NA & NA & 0.0 & 0.54 & $(0.27,0.81)$ & 3.94 & 0.00 & 13.7 & 1.28 & $(1.00,1.57)$ & 8.85 & 0.00 \\
\hline
\end{tabular}



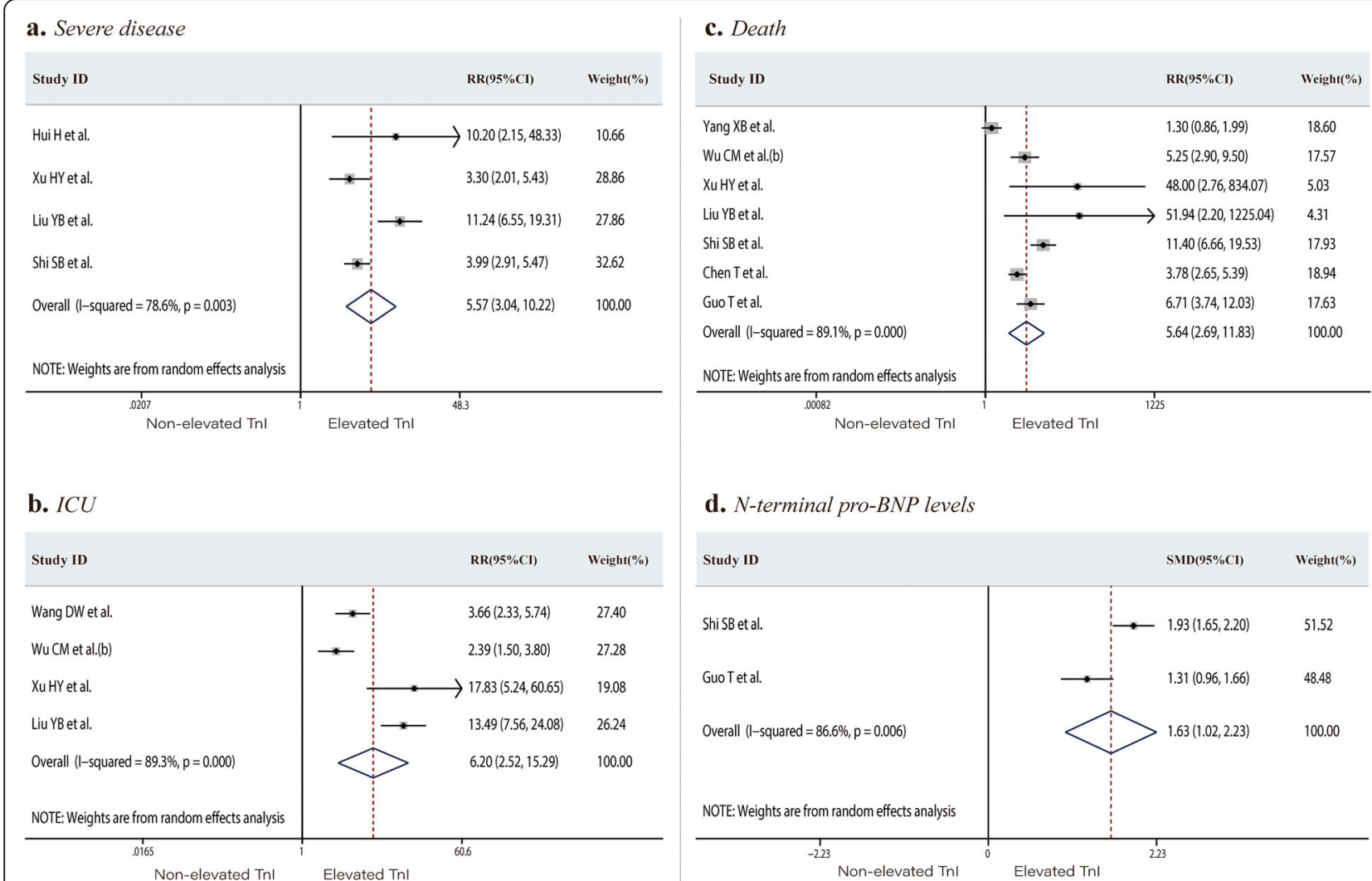

d. $N$-terminal pro-BNP levels

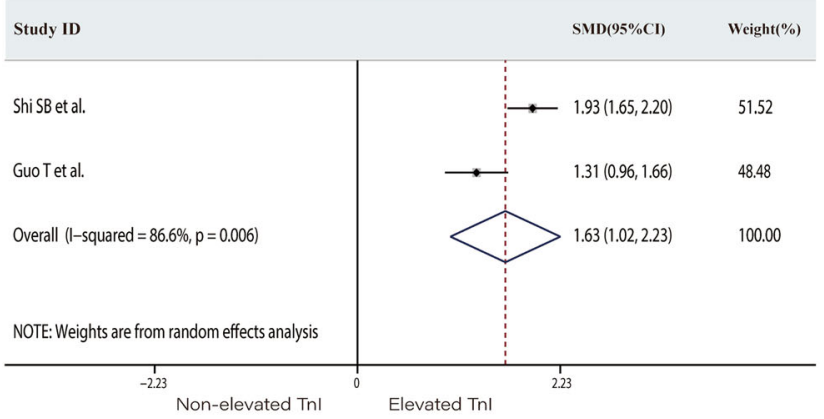

Fig. 2 Forest plots comparing of risk of severe disease (a), ICU admission (b), and death (c), and the N-terminal pro-BNP levels (d) in patients with or without elevated troponin I (or troponin T). ICU, intensive care unit; BNP, B-type natriuretic peptide; RR, risk ratios; SMD, standard mean

occurred at a rate of $11.9 \%$. Our analysis suggests that COVID-19 patients with elevated TnI levels are at higher risk of developing severe disease, requiring ICU admission, and death. Two studies from Wuhan (one with 416 cases, another with 187 cases) reported higher mortality among patients with cardiac injury than among those without $(51.2 \%$ vs. $4.5 \% ; P<0.001 ; 59.6 \%$ vs. $8.9 \%, P<0.001$, respectively) $[4,36]$. Patients with cardiac injury had higher serum concentrations of NTproBNP than those without cardiac injury [36, 39]. Patients with cardiac injury more commonly developed ARDS, were more likely to have ventricular tachycardia (VT) or ventricular fibrillation (VF), and had higher mortality rates than those without VT or VF $[36,39]$.

$\mathrm{TnI}$ has great prognostic significance for patients with COVID-19 as well as those with other influenza virus infections. In a study of 75 inpatients with SARS, acute myocardial infarction was the cause of 2 of 5 deaths [40]. Elevated TnI levels are also common in infections caused by other influenza virus subtypes [41-45]. TnI may play an important role in predicting the acute or long-term risk of influenza virus infection. Other biomarkers closely related to cardiac injury, such as CK, CK-MB, and LDH, were also selected in the meta- analysis. Our analysis showed that those with elevated $\mathrm{CK}, \mathrm{CK}-\mathrm{MB}$, and LDH were at a higher risk of developing severe disease or requiring ICU admission. The $\mathrm{LDH}$ level had a predictive value for death. Previous studies suggested that CK at ICU admission serves can be used as a biomarker of the severity of 2009 pandemic influenza A (pH1N1) infection [46].

Elevated TnI and CK-MB levels indicate cardiac injury such as viral myocarditis or myocardial infarction as well as multiple organ injury [47]. Initial reports showed that the possible pattern of myocardial injury is the early presentation of primary cardiovascular symptoms, as well as changes on echocardiography and ECG [3, 6, 4850]. Stress cardiomyopathy, supply demand mismatch (type II myocardial infarction), and myocarditis, sometimes similar to ST-segment elevation myocardial infarction, are all possible mechanisms [3, 6, 49]. In a study describing a single case without a history of cardiovascular disease, the patient had myocardial injury, and diffuse edema was seen on cardiac MRI [3]. Twelve lead ECG showed minimal diffuse ST-segment elevation and an ST-segment depression with T-wave inversion of lead $\mathrm{V} 1$ and aVR. Even in the absence of respiratory tract or infection symptoms, SARS-CoV-2 infection may cause 
a. Elevated TnI or TnT

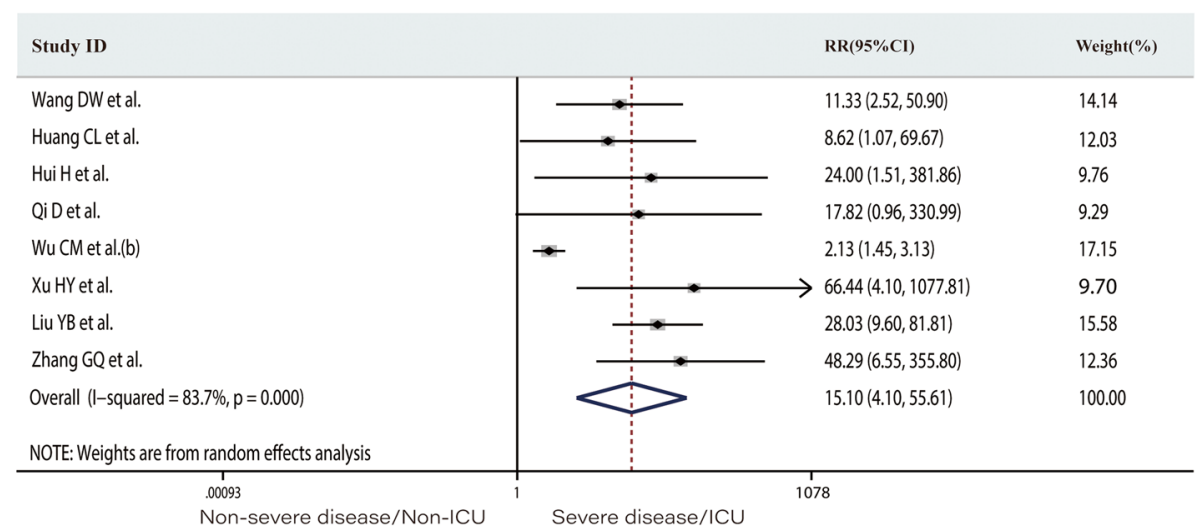

b. TnI levels

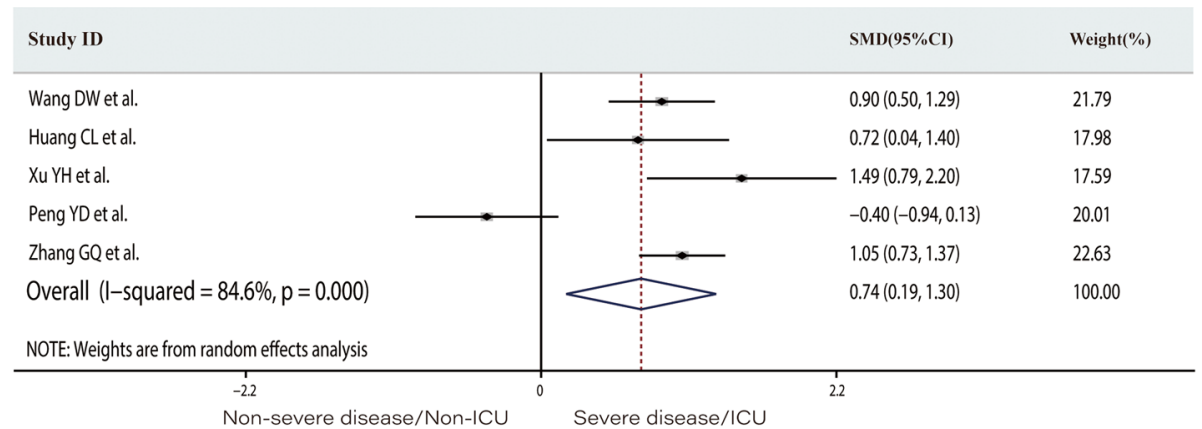

\section{c. Elevated TnI or TnT}

\begin{tabular}{|c|c|c|}
\hline Study ID & $\mathrm{RR}(\mathbf{9 5} \% \mathrm{CI})$ & Weight $(\%)$ \\
\hline Yang XB et al. & $1.88(0.58,6.11)$ & 13.55 \\
\hline Chen T et al. & $5.26(3.23,8.55)$ & 50.99 \\
\hline Guo Tet al. & $4.94(3.19,7.65)$ & 35.45 \\
\hline Overall $(I-$ squared $=22.5 \%, p=0.275)$ & $4.69(3.39,6.48)$ & 100.00 \\
\hline
\end{tabular}

Fig. 3 Forest plots comparing of the proportion of patients with elevated troponin I or troponin T levels in the severe disease/ICU group and in the non-severe disease/non-ICU group (a), the troponin I levels in the severe disease/ICU group and in the non-severe disease/non-ICU group (b), and the proportion of patients with elevated troponin I or troponin T levels in the survivors and non-survivors groups (c). ICU, intensive care unit; $\mathrm{RR}$, risk ratios; $\mathrm{SMD}$, standard mean

cardiac involvement. However, it is a pity that an endomyocardial biopsy was not performed; thus, there was no histological evidence [3]. Cardiac injury is an important prognostic factor for COVID-19. It is rational to presume that the virus affects the myocardium, and once patients develop severe pneumonia, cardiac injury or dysfunction is more likely to occur, leading to deterioration. In a study of critically ill patients, including 21 who had SARS-CoV-2 infection in the USA, the incidence of cardiomyopathy was high $(n=7$ [33\%]) [51]. In a patient without fever and respiratory symptoms, the initial ECG showed diffuse ST elevations and an admission TnI level of $7.9 \mathrm{ng} / \mathrm{mL}$, but angiography demonstrated non-obstructive coronary artery disease. After 


\section{a. Elevated $C K$}

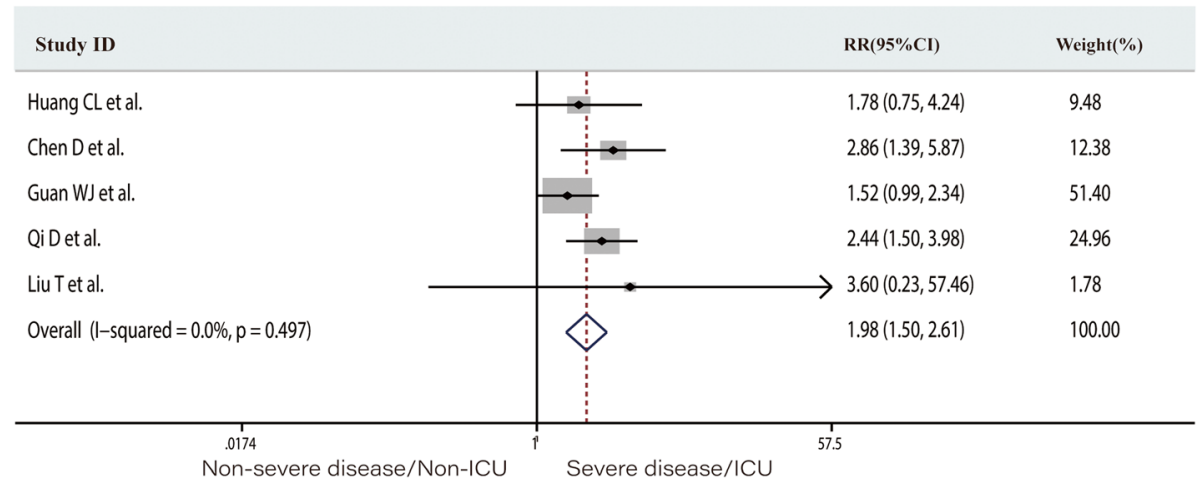

b. CK levels

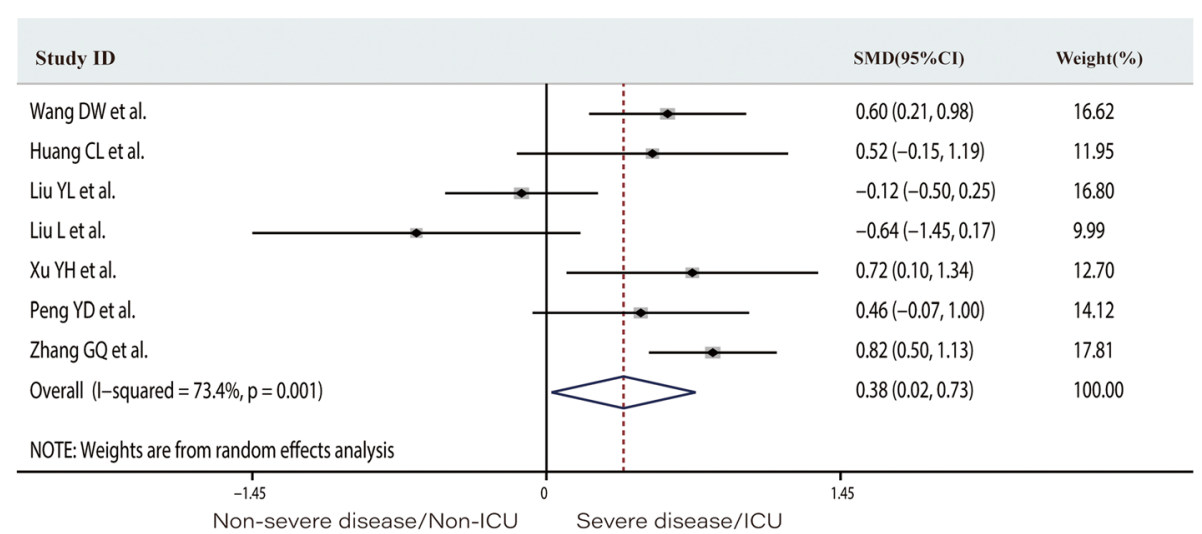

\section{c. Elevated $C K-M B$}

\begin{tabular}{|c|c|c|}
\hline Study ID & $\operatorname{RR}(95 \% \mathrm{CI})$ & Weight( $\%)$ \\
\hline Chen D et al. & $\rightarrow 6.23(3.50,11.08)$ & 32.14 \\
\hline QiD et al. & $2.82(1.51,5.28)$ & 30.77 \\
\hline Wu CM et al.(b) & $2.07(1.42,3.03)$ & 37.09 \\
\hline Overall $(1-$ squared $=79.8 \%, p=0.007)$ & $3.24(1.66,6.34)$ & 100.00 \\
\hline NOTE: Weights are from random effects analysis & & \\
\hline${ }_{.0903}^{1}$ & 11.1 & \\
\hline
\end{tabular}

Fig. 4 Forest plots comparing of the proportion of patients with elevated creatinine kinase levels in the severe disease/ICU group and in the non-severe disease/non-ICU group (a), the creatinine kinase levels in the severe disease/ICU group and in the non-severe disease/non-ICU group (b), and the proportion of patients with elevated creatinine kinase-myocardial band levels in the severe disease/ICU group and in the non-severe disease/non-ICU group (c). ICU, intensive care unit; RR, risk ratios; SMD, standard mean

treatment, this patient improved in the short term, but the long-term effects of myocardial injury remain to be determined [8].
The etiology of cardiac dysfunction may be multifactorial and related to infective myocarditis and/or ischemia. Pathological findings suggested a few interstitial 
a. Elevated $L D H$

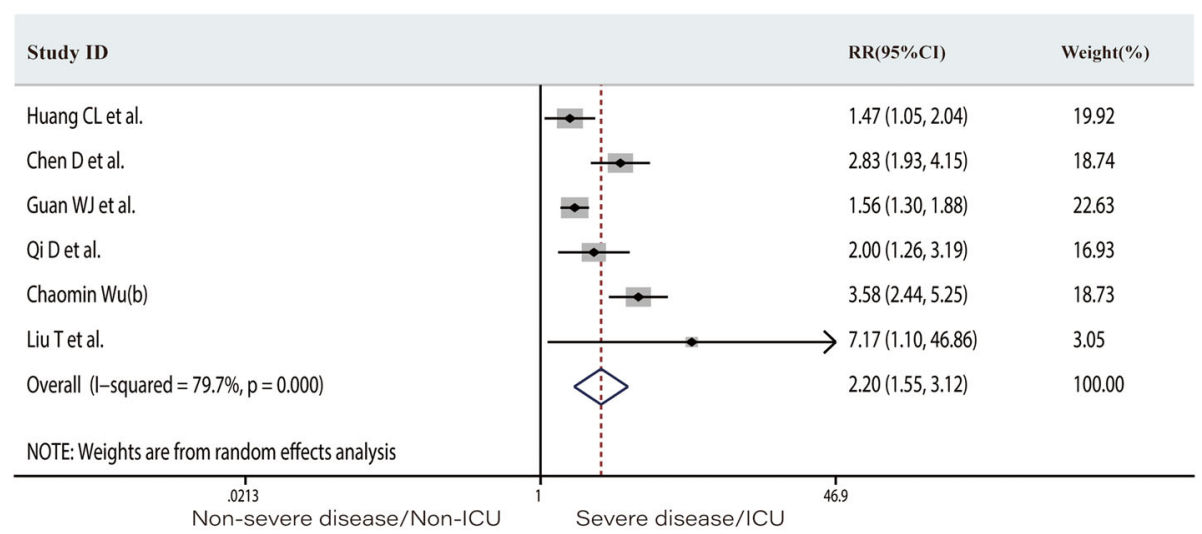

b. LDH levels

\begin{tabular}{|c|c|c|}
\hline Study ID & $\operatorname{SMD}(95 \% \mathrm{CI})$ & Weight $(\%)$ \\
\hline Wang DW et al. & $1.58(1.16,2.00)$ & 14.74 \\
\hline Wu CM et al.(a) & $1.29(0.98,1.59)$ & 15.49 \\
\hline Huang $\mathrm{CL}$ et al. & $1.01(0.31,1.70)$ & 12.57 \\
\hline Liu YL et al. & $0.99(0.60,1.39)$ & 14.92 \\
\hline Xu YH et al. & $1.12(0.49,1.76)$ & 13.08 \\
\hline Peng YD et al. & $-0.71(-1.25,-0.18)$ & 13.88 \\
\hline Zhang GQ et al. & $1.53(1.19,1.86)$ & 15.32 \\
\hline Overall (I-squared $=89.4 \%, p=0.000)$ & $0.99(0.48,1.50)$ & 100.00 \\
\hline NOTE: Weights are from random effects analysis & & \\
\hline-2 & & \\
\hline
\end{tabular}

\section{c. LDH levels}

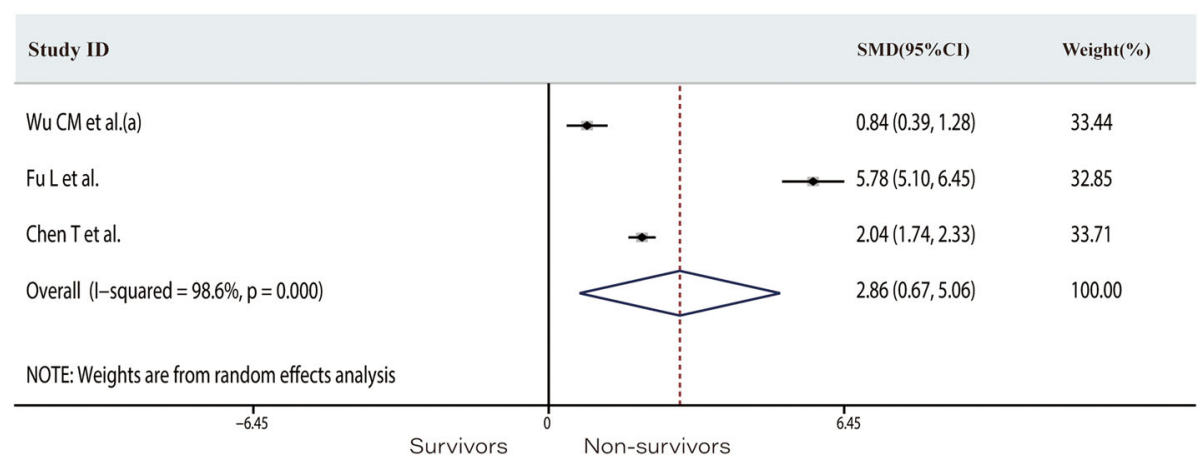

Fig. 5 Forest plots comparing of the proportion of patients with elevated lactate dehydrogenase levels in the severe disease/ICU group and in the non-severe disease/non-ICU group (a), the lactate dehydrogenase levels in the severe disease/ICU group and in the non-severe disease/nonICU group (b), and the lactate dehydrogenase levels in the survivors and non-survivor groups (c). ICU, intensive care unit; RR, risk ratios; SMD, standard mean

mononuclear inflammatory infiltrates in the myocardial interstitial [52]. Viral invasion may cause direct cardiac injury, and COVID-19-induced cytokine storm may also have toxic effects on the myocardium [53]. Cytokine storm may play a role in the development of ARDS and fulminant myocarditis. In our analysis, 3 studies reported the laboratory findings of IL- 6 levels in 526 patients. IL6 levels were significantly higher in the severe disease/ 


\section{a. Arrhythmia}

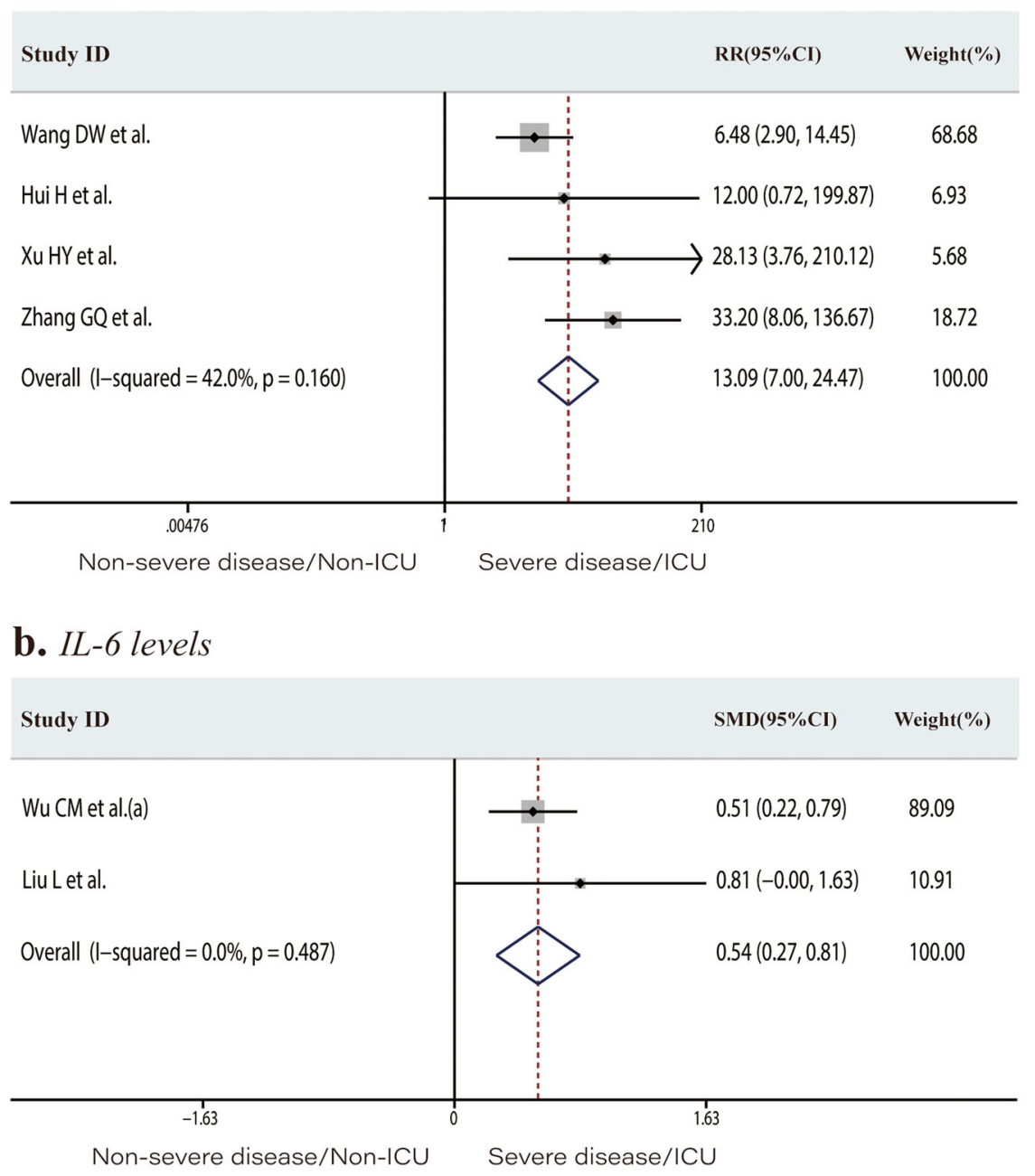

\section{c. IL-6 levels}

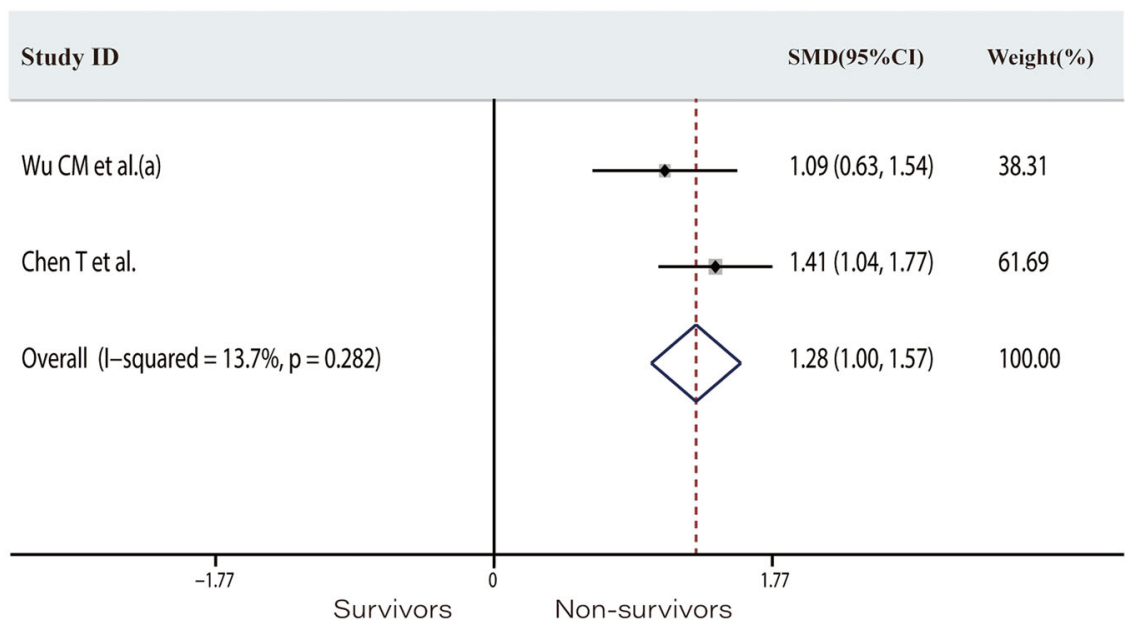

Fig. 6 Forest plots comparing of the risk of developing to severe disease or requiring ICU admission among patients with or without newly occurring arrhythmias (a), the interleukin-6 levels in the severe disease/ICU group (b), and in the non-severe disease/non-ICU group and the interleukin-6 levels in the survivors and non-survivors groups (c). ICU, intensive care unit; RR, risk ratios; SMD, standard mean 
ICU groups than non-severe disease/non-ICU groups, as well as in non-survivors than in survivors. Cardiac involvement reportedly occurred a few days after the influenza syndrome, suggesting the mechanisms of a potential myocyte dissemination of the virus activating the immune system, eventually leading to the onset of heart failure [3]. A study reported that, compared to survivors, non-survivors had increased concentrations of $\mathrm{C}$ reactive protein, decreased lymphocyte counts, and significantly reduced numbers of $\mathrm{CD} 3+\mathrm{CD} 8+\mathrm{T}$ cells, resulting in an immune response [54]. Anti-IL-6, as a drug targeting cytokine pathway and based on its mechanism of action, has potential benefits in COVID-19related ARDS and pneumonia [55].

\section{Arrhythmia}

Our analysis also found that the patients with emerging arrhythmia are at a higher risk of developing severe disease or requiring ICU admission. In a study of 41 patients with COVID-19, atrial fibrillation occurred in 2 of 3 severe and critical patients with tachycardia, with a peak heart rate of $160 \mathrm{bpm} \mathrm{[22].} \mathrm{Newly} \mathrm{occurring} \mathrm{arrhythmias} \mathrm{are}$ often closely related to cardiac injury. The incidence of ventricular arrhythmias (VT/VF) among 187 patients with COVID-19 was $5.9 \%$, primarily affecting those with elevated cardiac troponin levels [36]. One study reported that 5 of 6 acute myocardial injury patients had more than two kinds of ECG abnormalities, including ST-T/Q curve abnormality, atrioventricular block, and arrhythmia [28]. Severe pneumonia increases the resistance of the pulmonary circulation, increasing the pressure of the right atrium, and leading to atrial tachyarrhythmia. Antiviral drugs such as hydroxychloroquine may also prolong the QT interval. Alternatively, the virus directly damages the myocardium and the cardiac conduction system, causing multiple ventricular premature and atrioventricular block. More attention is needed on arrhythmia among severe disease/ICU admission COVID-19 patients. However, in the studies reviewed here, ECG or echocardiography was rarely performed and the occurrence of arrhythmia was rarely reported.

\section{Conclusion}

This meta-analysis included the largest sample size and is the first to analyze the correlation of cardiac injury biomarkers and arrhythmia with mortality and other prognosis. Our systematic review and meta-analysis indicate that patients with elevated TnI (TnT) levels are at significantly higher risk of developing severe disease, requiring ICU admission, or death. Our analysis also reveals that patients with elevated CK, CK-MB, and LDH levels and emerging arrhythmia were at a higher risk of developing severe disease, requiring ICU admission. LDH levels also have predictive value for death.
Therefore, we strongly recommend the close monitoring of cardiac injury-related biomarkers in COVID-19 patients, especially during the acute disease phase.

\section{Limitations and prospects}

The current clinical attention given to cardiac injury may be insufficient, and the strong infection of the virus makes cardiovascular examinations such as MRI, echocardiography, and coronary angiography difficult to perform [56]. The evaluation of cardiac injury biomarkers combined with cardiac examinations may help better assessments of the condition. There are few reports on cardiac injury in COVID-19 patients, and a large amount of evidence is still needed to make the necessary risk predictions and stratifications. The present results provide some evidence for COVID-19 treatment guidelines. In the future, it well be necessary to strengthen the monitoring of cardiac injury biomarkers, combined with echocardiography [57], ECG, MRI, and other cardiac examinations, in patients with severe SARS-CoV-2 infection. When circulation support is needed in severe COVID-19 cases, the use of an intra-aortic balloon pump or extracorporeal membrane oxygenation may be considered [8].

\section{Supplementary information}

Supplementary information accompanies this paper at https://doi.org/10. 1186/s13054-020-03183-z.

Additional file 1: Table S1. Clinical characteristics of patients with COVID-19.

\section{Abbreviations \\ 2019-nCoV: Novel coronavirus; ARDS: Acute respiratory distress syndrome; $\mathrm{Cl}$ : Confidence interval; CK: Creatinine kinase; CK-MB: Creatinine kinase- myocardial band; COVID-19: Coronavirus disease; Hs-Tnl: High-sensitivity troponin I; ICU: Intensive care unit; IL-6: Interleukin-6; IQR: Interquartile range; LDH: Lactate dehydrogenase; MC: Multicenter study; $n$ : Number; NA: Not available; NCIP: Novel coronavirus-infected pneumonia; NT-proBNP: N- terminal pro-B-type natriuretic peptide; RT-PCR: Reverse transcriptase polymerase chain reaction; SARS-CoV-2: Severe acute respiratory syndrome coronavirus-2; SC: Single-center study; SD: Standard deviation; Tnl: Troponin I; TnT: Troponin T; TnT-HSST: Troponin T-hypersensitivity}

\section{Acknowledgements}

None.

\section{Authors' contributions}

$X \mathrm{~L}, \mathrm{HS}$, and $\mathrm{YWX}$ had full access to all of the data in the study and take responsibility for the integrity of the data and the accuracy of the data analysis. $X L, X P, Y L, N A, H S$, and $Y W X$ designed the study, searched the scientific literature, collected the data, and performed statistical analyses. $\mathrm{XL}$, $Y L, N A$, and $Y W X$ drafted the manuscript. $X L, X P, Y L$, and $H S$ participated in the design and resolved differences. HS and $Y W X$ contributed to the conception and quality assessment of the study. YFX, FY, LT, JS, YG, and HS helped to revise the manuscript and provided technical or material support. The authors have read and approved the final manuscript.

\section{Funding}

This work was supported by the National Key R\&D Program of China (grants 2018YFC1704900 \& 2018YFC1704901). 


\section{Availability of data and materials}

All the data supporting the conclusions of this article are included within the article.

\section{Ethics approval and consent to participate}

This article is meta-analysis and does not require ethics committee approval or a consent statement.

\section{Consent for publication}

All authors have agreed to the publication of this manuscript.

\section{Competing interests}

The authors declare that they have no competing interests.

\section{Author details}

'Guang'anmen Hospital, China Academy of Chinese Medical Sciences, Beijing, China. ${ }^{2}$ Beijing University of Chinese Medicine, Beijing, China. ${ }^{3}$ Institute of Basic Research In Clinical Medicine, China Academy Of Chinese Medical Sciences, Beijing, China. ${ }^{4}$ Key Laboratory of Chinese Internal Medicine of the Ministry of Education, Dongzhimen Hospital Affiliated to Beijing University of Chinese Medicine, Beijing, China. ${ }^{5}$ Shanxi University of Chinese Medicine, Taiyuan, China.

\section{Received: 30 April 2020 Accepted: 15 July 2020}

Published online: 28 July 2020

\section{References}

1. Coronavirus disease (COVID-19) Pandemic. Accessed 25 Apr 2020. https:// www.who.int/emergencies/diseases/novel-coronavirus-2019.

2. Huang $C L$, Wang $Y M$, Li XW, et al. Clinical features of patients infected with 2019 novel coronavirus in Wuhan, China. Lancet. 2020;395(10223):497-506.

3. Inciardi RM, Lupi L, Zaccone G, Italia L, Raffo M, Tomasoni D, et al. Cardiac involvement in a patient with coronavirus disease 2019 (COVID-19). JAMA Cardiol. 2020.

4. Shi SB, Qin M, Shen B, et al. Association of cardiac injury with mortality in hospitalized patients with COVID-19 in Wuhan, China JAMA Cardiol Published online March 25, 2020.

5. Deng G, Yin M, Chen X, Zeng F. Clinical determinants for fatality of 44,672 patients with COVID-19. Critical care (London, England). 2020;24(1):179.

6. Cortese B. COVID-19 pandemic-some cardiovascular considerations from the trench. Am Heart J. 2020;225:1-2.

7. Loungani RS, Rehorn MR, Newby LK, Katz JN, Klem I, Mentz RJ, Jones WS, Vemulapalli S, Kelsey AM, Blazing MA, et al. A care pathway for the cardiovascular complications of COVID-19: insights from an institutional response. Am Heart J. 2020;225:3-9.

8. Fried JA, Ramasubbu K, Bhatt R, Topkara VK, Clerkin KJ, Horn E, et al. The variety of cardiovascular presentations of COVID-19. Circulation. 2020; 141(23):1930-6.

9. Madjid M, Safavi-Naeini P, Solomon SD, Vardeny O. Potential effects of coronaviruses on the cardiovascular system: a review. JAMA Cardiol. 2020.

10. Hutton B, Salanti G, Caldwell DM, Chaimani A, Schmid CH, Cameron C, et al. The PRISMA extension statement for reporting of systematic reviews incorporating network meta-analyses of health care interventions: checklist and explanations. Ann Intern Med. 2015;162(11):777-84.

11. World Health Organization. Clinical management of severe acute respiratory infection when novel coronavirus ( $\mathrm{nCoV}$ ) infection is suspected: interim guidance. Published January 28, 2020. Accessed April 5, 2020. https://www. who.int/publications-detail/clinical-managementof-severe-acute-respiratoryinfection-when-novelcoronavirus-(ncov)-infection-is-suspected.

12. Hozo SP, Djulbegovic B, Hozo I. Estimating the mean and variance from the median, range, and the size of a sample. BMC Med Res Methodol. 2005;5:13.

13. Stang A. Critical evaluation of the Newcastle-Ottawa scale for the assessment of the quality of nonrandomized studies in meta-analyses. Eur J Epidemiol. 2010;25(9):603-5.

14. Sterne JA, Gavaghan D, Egger M. Publication and related bias in metaanalysis: power of statistical tests and prevalence in the literature. J Clin Epidemiol. 2000;53(11):1119-29.

15. Leung WK, Ho HJ, Lin JT, Wu MS, Wu CY. Prior gastroscopy and mortality in patients with gastric cancer: a matched retrospective cohort study. Gastrointest Endosc. 2018;87(1):119-27 e3.
16. Wang DW, Hu B, Hu C, et al. Clinical characteristics of 138 hospitalized patients with 2019 novel coronavirus-infected pneumonia in Wuhan, China JAMA Published online February 7, 2020.

17. Wu CM, Chen XY, Cai YP, et al. Risk factors associated with acute respiratory distress syndrome and death in patients with coronavirus disease 2019 pneumonia in Wuhan, China JAMA Intern Med Published online March 13, 2020.

18. Yang $X B, Y u Y, X U$ JQ, et al. Clinical course and outcomes of critically ill patients with SARS-CoV-2 pneumonia in Wuhan, China: a single-centered, retrospective, observational study. Lancet Respir Med. 2020;8(5):475-81.

19. Chen D, Li XK, Song QF, Hu CC, Su FF, Dai JY. Hypokalemia and clinical implications in patients with coronavirus disease 2019 (COVID-19). Published 2020. Accessed 19 Mar 2020. https://www.medrxiv.org/content/10.1101/202 0.02.27.20028530v1.

20. Fu L, Fei J, Xiang HX, et al. Influence factors of death risk among COVID-19 patients in Wuhan, China: a hospital-based case-cohort study. Published 2020. Accessed 18 Mar 2020. https://www.medrxiv.org/content/10.1101/202 $0.03 .13 .20035329 \mathrm{v} 1$.

21. Guan WJ, Ni ZY, Hu Y, Liang WH, Ou CQ, He JX, et al. Clinical characteristics of coronavirus disease 2019 in China. N Engl J Med. 2020:382(18):1708-20.

22. Hui $H$, Zhang $Y Q$, Yang $X$, et al. Clinical and radiographic features of cardiac injury in patients with 2019 novel coronavirus pneumonia. Published 2020. Accessed 19 Mar 2020. https://www.medrxiv.org/content/10.1101/2020. 02.24.20027052v1.

23. Liu YL, Sun WW, Li J, et al. Clinical features and progression of acute respiratory distress syndrome in coronavirus disease 2019. Published 2020. Accessed 19 Mar 2020. https://www.medrxiv.org/content/10.1101/2020.02.1 $7.20024166 \mathrm{v} 3$.

24. Liu L, Gao JY. Clinical characteristics of 51 patients discharged from hospital with COVID-19 in Chongqing, China. Published 2020. Accessed 18 Mar 2020 https://www.medrxiv.org/content/10.1101/2020.02.20.20025536v1.

25. Qi D, Yan XF, Tang XM, et al. Epidemiological and clinical features of 2019$n C o V$ acute respiratory disease cases in Chongqing municipality, China: a retrospective, descriptive, multiple-center study. Published 2020. Accessed 21 Mar 2020. https:/www.medrxiv.org/content/10.1101/2020.03.01.20029397v1.

26. Wang YF, Zhou Y, Yang Z, Xia DP, Geng S. Clinical characteristics of patients with severe pneumonia caused by the 2019 novel coronavirus in Wuhan, China. Published 2020. Accessed 21 Mar 2020. https://www.medrxiv.org/ content/10.1101/2020.03.02.20029306v1.

27. $\mathrm{Wu} \mathrm{CM}$, Hu XL, Song JX, et al. Heart injury signs are associated with higher and earlier mortality in coronavirus disease 2019 (COVID-19). Published 2020. Accessed 23 Mar 2020. https://www.medrxiv.org/content/10.1101/202 $0.02 .26 .20028589 \mathrm{v} 1$.

28. $\mathrm{Xu} \mathrm{HY}, \mathrm{Hou} \mathrm{KK}, \mathrm{Xu} \mathrm{H}$, et al. Acute myocardial injury of patients with coronavirus disease 2019. Published 2020. Accessed 23 Mar 2020. https:// www.medrxiv.org/content/10.1101/2020.03.05.20031591v1.

29. $\mathrm{Xu} Y H, X u Z H, L i u X S$, et al. Clinical findings in critical ill patients infected with SARS-Cov-2 in Guangdong Province, China: a multi-center, retrospective, observational study. Published 2020. Accessed 20 Mar 2020. https://www.medrxiv.org/content/10.1101/2020.03.03.20030668v1.

30. Liu YB, Li JL, Liu DH, et al. Clinical features and outcomes of 2019 novel coronavirus-infected patients with cardiac injury. Published 2020. Accessed 21 Mar 2020. https:/www.medrxiv.org/content/10.1101/2020.03.11.20030957v1.

31. Peng YD, Meng K, Guan HQ, et al. Clinical characteristics and outcomes of 112 cardiovascular disease patients infected by 2019-nCoV. Zhonghua Xin Xue Guan Bing Za Zhi. 2020;48(0):E004.

32. Zhang GQ, Hu C, Luo LJ, et al. Clinical features and outcomes of 221 patients with COVID-19 in Wuhan, China. Published 2020. Accessed 16 Mar 2020. https://www.medrxiv.org/content/10.1101/2020.03.02.20030452v1.

33. Liu T, Zhang JY, Yang YH, et al. The potential role of IL-6 in monitoring severe case of coronavirus disease 2019. Published 2020. Accessed 22 Mar 2020. https://www.medrxiv.org/content/10.1101/2020.03.01.20029769v2.

34. Wu J, Li W, Shi XW, et al. Early antiviral treatment contributes to alleviate the severity and improve the prognosis of patients with novel coronavirus disease (COVID-19). J Intern Med. 2020:288(1):128-38.

35. Chen T, Wu D, Chen HL, et al. Clinical characteristics of 113 deceased patients with coronavirus disease 2019: retrospective study. BMJ. 2020;368: m1091.

36. Guo T, Fan YZ, Chen M, et al. Cardiovascular implications of fatal outcomes of patients with coronavirus disease 2019 (COVID-19). JAMA Cardiol. 2020; https://doi.org/10.1001/jamacardio.2020.1017. 
37. Ndrepepa G, Kastrati A. Creatine kinase myocardial band - a biomarker to assess prognostically relevant periprocedural myocardial infarction. Int J Cardiol. 2018;270:118-9.

38. Vasudevan G, Mercer DW, Varat MA. Lactic dehydrogenase isoenzyme determination in the diagnosis of acute myocardial infarction. Circulation. 1978;57(6):1055-7.

39. Lippi G, Lavie CJ, Sanchis-Gomar F. Cardiac troponin I in patients with coronavirus disease 2019 (COVID-19): evidence from a meta-analysis. Prog Cardiovasc Dis. 2020.

40. Peiris JS, Chu CM, Cheng VC, Chan KS, Hung IF, Poon LL, Law KI, Tang BS, Hon TY, Chan CS, et al. Clinical progression and viral load in a community outbreak of coronavirus-associated SARS pneumonia: a prospective study. Lancet (London, England). 2003;361(9371):1767-72.

41. Erden I, Erden EC, Ozhan H, Basar C, Yildirim M, Yalçin S, Aydin LY, Dumlu T. Echocardiographic manifestations of pandemic 2009 (H1N1) influenza a virus infection. J Infection. 2010;61(1):60-5.

42. Haessler S, Paez A, Rothberg M, Higgins T. 2009 pandemic H1N1-associated myocarditis in a previously healthy adult. Clin Microbiol Infection. 2011;17(4):572-4.

43. Chacko B, Peter JV, Pichamuthu K, Ramakrishna K, Moorthy M, Karthik R, John G. Cardiac manifestations in patients with pandemic (H1N1) 2009 virus infection needing intensive care. J Critical Care. 2012;27(1):106 e101-106.

44. Kumar K, Guirgis M, Zieroth S, Lo E, Menkis AH, Arora RC, Freed DH. Influenza myocarditis and myositis: case presentation and review of the literature. Can J Cardiol. 2011;27(4):514-22.

45. Ito T, Akamatsu K, Ukimura A, Fujisaka T, Ozeki M, Kanzaki Y, Ishizaka N: The Prevalence and findings of subclinical influenza-associated cardiac abnormalities among Japanese Patients. Internal Med (Tokyo, Japan) 2018, 57(13):1819-1826.

46. Borgatta B, Pérez M, Rello J, Vidaur L, Lorente L, Socías L, Pozo JC, Pozo J, Garnacho-Montero J, Rello J. Elevation of creatine kinase is associated with worse outcomes in 2009 pH1N1 influenza A infection. Intensive Care Med. 2012;38(7):1152-61.

47. O'Brien PJ. Cardiac troponin is the most effective translational safety biomarker for myocardial injury in cardiotoxicity. Toxicology. 2008;245(3):206-18.

48. Hu H, Ma F, Wei X, Fang Y. Coronavirus fulminant myocarditis saved with glucocorticoid and human immunoglobulin. Eur Heart J. 2020.

49. Sala S, Peretto G, Gramegna M, Palmisano A, Villatore A, Vignale D, De Cobelli F, Tresoldi M, Cappelletti AM, Basso C, et al. Acute myocarditis presenting as a reverse Tako-Tsubo syndrome in a patient with SARS-CoV-2 respiratory infection. Eur Heart J. 2020;41(19):1861-2.

50. Zeng JH, Liu YX, Yuan J, Wang FX, Wu WB, Li JX, Wang LF, Gao H, Wang Y, Dong CF, et al. First case of COVID-19 complicated with fulminant myocarditis: a case report and insights. Infection. 2020:1-5.

51. Arentz M, Yim E, Klaff L, Lokhandwala S, Riedo FX, Chong M, et al. Characteristics and outcomes of 21 critically ill patients with COVID-19 in Washington state. Jama. 2020.

52. Xu Z, Shi L, Wang Y, Zhang J, Huang L, Zhang C, et al. Pathological findings of COVID-19 associated with acute respiratory distress syndrome. Lancet Respir Med. 2020;8(4):420-2.

53. Clerkin KJ, Fried JA, Raikhelkar J, Sayer G, Griffin JM, Masoumi A, et al. Coronavirus disease 2019 (COVID-19) and cardiovascular disease. Circulation. 2020;141(20):1648-55.

54. Du RH, Liang LR, Yang CQ, Wang W, Cao TZ, Li M, et al. Predictors of mortality for patients with COVID-19 pneumonia caused by SARS-CoV-2: a prospective cohort study. Eur Respir J. 2020;55(5):2000524.

55. Convertino I, Tuccori M, Ferraro S, Valdiserra G, Cappello E, Focosi D, Blandizzi C. Exploring pharmacological approaches for managing cytokine storm associated with pneumonia and acute respiratory distress syndrome in COVID-19 patients. Critical Care (London, England). 2020;24(1):331.

56. Welt FGP, Shah PB, Aronow HD, Bortnick AE, Henry TD, Sherwood MW, et al. Catheterization laboratory considerations during the coronavirus (COVD-19) pandemic: from ACC's interventional council and SCAI. J Am Coll Cardiol. 2020.

57. Peng QY, Wang XT, Zhang LN. Using echocardiography to guide the treatment of novel coronavirus pneumonia. Critical Care (London, England). 2020:24(1):143

\section{Publisher's Note}

Springer Nature remains neutral with regard to jurisdictional claims in published maps and institutional affiliations.

Ready to submit your research? Choose BMC and benefit from:

- fast, convenient online submission

- thorough peer review by experienced researchers in your field

- rapid publication on acceptance

- support for research data, including large and complex data types

- gold Open Access which fosters wider collaboration and increased citations

- maximum visibility for your research: over $100 \mathrm{M}$ website views per year

At BMC, research is always in progress.

Learn more biomedcentral.com/submissions 